Numerical Methods for the Simulation of Flow in Root-Soil Systems

\author{
Todd Arbogast \\ Mandri Obeyesekere \\ Mary F. Wheeler
}

April 1991

TR91-07 



\title{
NUMERICAL METHODS FOR THE SIMULATION OF FLOW IN ROOT-SOIL SYSTEMS*
}

\author{
TODD ARBOGAST $\dagger$, MANDRI OBEYESEKERE‡, AND MARY F. WHEELER $\dagger$
}

\begin{abstract}
We consider the numerical properties of approximation schemes for a model that simulates water transport in root-soil systems. The model given in this paper is a reformulation of a previously proposed model now defined completely in terms of the water potential. The system of equations consists of a parabolic partial differential equation which contains a nonlinear capacity term coupled to two linear ordinary differential equations. A closed form solution is obtained for one of the latter equations. Finite element and finite difference schemes are defined to approximate the solution of the coupled system, and optimal order error estimates are derived. A postprocessed water mass flux computation is also presented and shown to be superconvergent to the true flux. Computational results which verify the theoretical convergence rates are given.
\end{abstract}

Key words. error estimates, finite elements, finite differences, fluxes, root-soil, nonlinear, Richard's equation

AMS(MOS) subject classifications. 65M15, 65M60, 76S05, 92-08, 92B99

1. Introduction. It is well known that due to transpiration of water through plant leaves, soil-water flows through soil into a plant's root system, and then through its xylem vessels up to its leaves. One objective of agricultural engineers is to produce new varieties of plants that either lose less water through transpiration or make better use of it and are thereby better suited to dry climates. One method currently used to regulate a plant's consumption of soil-water is to change the characteristics of the cells of its root systern. Therefore, when simulating the water uptake of a root-soil system, it is important to use a model that incorporates the effects of the root's different cell layers on water transport. Such a model was given by Obeyesekere in [15], and it applies to plants possessing taproots (e.g., cotton plants). Most of the models preceding this one merely incorporated the water uptake effect through an ernpirically defined source function (see, e.g., [14] and [12]), although the work of [11] and [13] moves in the direction of actual flow modeling.

Water transport in a root-soil system is due mainly to the water potential gradient created by transpiration itself. Thus, we choose to consider a form of the model which describes the behavior of the water potential in both the soil and root's cells for given transpiration and evaporation rates. As described in [15], the macroscopic model describes the vertical flow of soil-water through the soil matric where the root's effects are incorporated in a sink term. In the original form of the model, an ordinary differential equation was given which describes the water flow rate in the taproot xylem bundles. We reformulate this part of the model in terms of the water potential in the taproot xylems. Connecting the two parts of the macroscopic model is a microscopic model similar to that in [15] which describes the flow around a generic subroot. The full model then consists of three second order differential equations: a nonlinear parabolic partial differential equation coupled to two linear ordinary differential equations, one governing the vertical (axial) flow of water through the taproots

*This work was supported in part by the National Science Foundation and the U.S. Department of Energy.

†Department of Mathematical Sciences, Rice University, Houston, Texas 77251

$\ddagger$ Department of Mathematics, University of Houston, Houston, Texas 77204 
and the other (microscopic model) which describes the radial flow around a root and gives the resulting extraction of water by the plant.

In the next section we present the reformulated model. In Section 3 we describe a general finite element approximation scheme for the model. We then show that this scheme's solution converges to the true solution at the optimal rate in Section 4. In Section 5, we improve the approximation of the water mass fluxes by a simple postprocessing procedure, and we show that this approximate flux is superconvergent to the true flux. Section 6 deals with a finite difference scheme, and again optimal rates of convergence are shown and superconvergent fluxes are given. In the last section, we present some numerical results which substantiate the rates of convergence of the finite difference scheme.

In a related paper [4], the authors discuss the effects of various genetic and physical parameters on plant performance based on the predictions of this model approximated by the finite difference scheme given here.

2. The reformulated model. We first consider the flow of water in the soil. It is assumed that the block of soil containing the roots (see Fig. 1) is an unsaturated porous medium and that the macroscopic flow is one dimensional, being in the vertical direction only. Moreover, we assume that the mass of water extracted by the subroots is macroscopically distributed throughout the medium. Suppose that the block of soil containing the root system is a cylinder of depth $L$ and cross-sectional area $A_{s}$. Conservation of water mass combined with Darcy's law for vertical flow in soil gives Richard's equation [10] for the matric potential $\psi_{s}(z, t)$. If $\rho$ is the density of water, $\theta(\psi)$ is the water content (volume of water per unit volume of soil), $K_{s}(z, \psi)$ is the conductivity of water in the soil (permeability of water times $\rho^{2}$ divided by the water viscosity), and $g$ is the gravitational acceleration constant, then

$$
\rho \frac{\partial \theta\left(\psi_{s}\right)}{\partial t}-\frac{\partial}{\partial z}\left(K_{s}\left(\psi_{s}\right)\left(\frac{\partial \psi_{s}}{\partial z}-g\right)\right)=-A_{s}^{-1} \mathcal{S} \text { for } z \in I, t \in J
$$

where $\mathcal{S}$ represents the macroscopic extraction of water by the plant's roots (defined by (2.11) or (2.13) below), $I=(0, L), J=(0, T]$, and $T$ is some final time. To this equation we impose a Neumann boundary condition to model evaporation and precipitation at the surface of the ground where $z=0$, a Dirichlet condition to model continuity of potential at the lower end $z=L$ (we would probably assume that $L$ is the depth of the water table, so then the Dirichlet condition reflects saturated conditions), and an initial condition:

$$
\begin{aligned}
& K_{s}\left(0, \psi_{s}(0, t)\right)\left(\frac{\partial \psi_{s}}{\partial z}(0, t)-g\right)=q_{\mathrm{evap}}\left(t, \psi_{s}(0, t)\right)-q_{\mathrm{prec}}(t) \text { for } t \in J, \\
& \psi_{s}(L, t)=\psi_{\mathrm{bot}}(t) \text { for } t \in J \\
& \psi_{s}(z, 0)=\psi_{s}^{0}(z) \text { for } z \in I .
\end{aligned}
$$

For consistency, $\psi_{s}^{0}(L)=\psi_{\text {bot }}(0)$.

For the taproot, we assume that the taproot xylem bundles form a one dimensional saturated porous medium that contains a distributed water source term. Conservation of water implies that this source term is the same as the sink term for the flow in the soil medium. Again it is Richard's equation that governs the water potential in the taproot xylems, $\psi_{x}(z, t)$. If $A_{x}(z, t)$ is the total cross-sectional area of the xylems in 


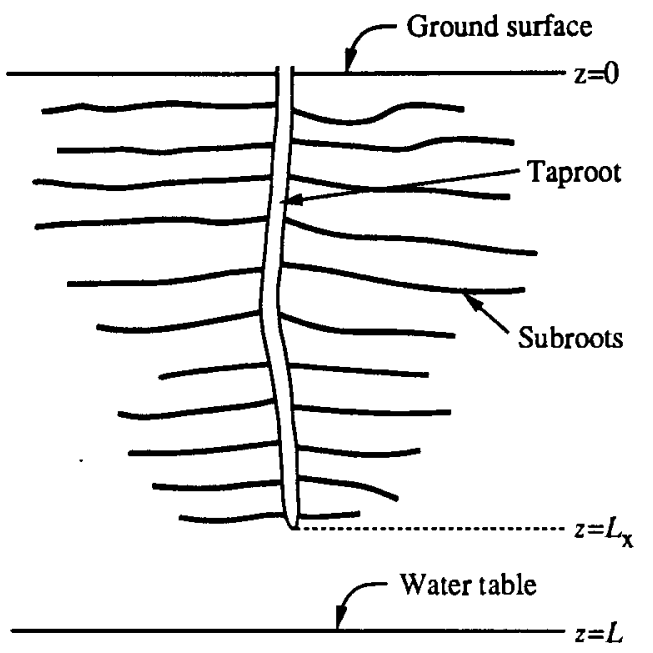

Fig. 1. A root-soil system.

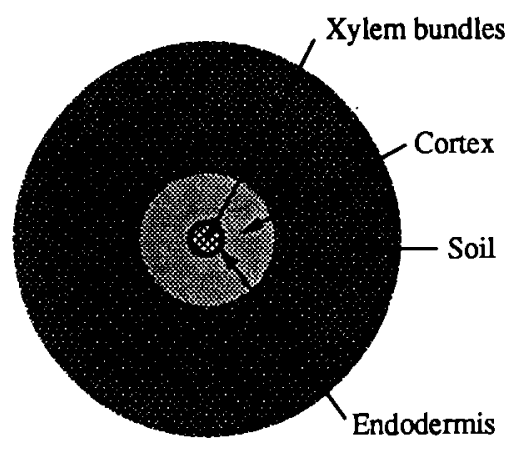

Fig. 2. Cross-section of a root.

the taproot, $L_{x}(t) \leq L$ is its length, and $\bar{K}_{x}(z, t)$ is its conductivity under saturated conditions, then we have that the axial flow through the taproot is given by

$$
-\frac{\partial}{\partial z}\left(\bar{K}_{x}\left(\frac{\partial \psi_{x}}{\partial z}-g\right)\right)=A_{x}^{-1} \mathcal{S} \quad \text { for } z \in I_{x}(t), t \in J
$$

where $I_{x}(t)=\left(0, L_{x}(t)\right)$. To this equation we impose a Neumann boundary condition representing the given transpiration rate at ground level and a no-flow condition at the lower end of the taproot:

$$
\begin{aligned}
& \bar{K}_{x}(0, t)\left(\frac{\partial \psi_{x}}{\partial z}(0, t)-g\right)=A_{x}^{-1}(0, t) q_{\operatorname{trans}}(t) \quad \text { for } t \in J \\
& \bar{K}_{x}\left(L_{x}(t), t\right)\left(\frac{\partial \psi_{x}}{\partial z}\left(L_{x}(t), t\right)-g\right)=0 \quad \text { for } t \in J
\end{aligned}
$$

The coupling term $\mathcal{S}$ is calculated using the microscopic model. In the microscopic model, we consider saturated porous medium flow through the subroot cortex as well as through a small cylinder of soil around the subroot (see Fig. 2). The permeability of the medium changes as we go from the soil to the cortex, and a resistance to flow is encountered at the endodermis. In this model it is assumed that at a given depth, the water potential in the xylems of the taproot and the xylems of a subroot are equal (i.e., that the resistance to axial flow in the subroot is negligible). Neglecting the effects of gravity, then, the microscopic flow is restricted to the radial direction. So we consider the flow only in a cross-section of a generic subroot at depth $z$ and time $t$. Again Richard's equation governs the water potential $\psi_{r}(z, r, t)$ in the subroot; for each fixed $z \in I_{x}(t)$ and $t \in J$, in cylindrical coordinates this is

$$
-\frac{\partial}{\partial r}\left(r K_{r}(z, r, t) \frac{\partial \psi_{r}}{\partial r}\right)=0 \quad \text { for } r_{e}(z, t)<r<r_{s}(z, t),
$$

where $r_{e}(z, t)$ and $r_{s}(z, t)$ are the radii of the endodermis and of the saturated soil cylinder around the subroot, respectively, and $K_{r}(z, r, t)$ is the conductivity. This function is

$$
K_{r}(z, r, t)= \begin{cases}\bar{K}_{s}(z) & \text { if } r_{c}(z, t) \leq r \leq r_{s}(z, t) \\ \bar{K}_{c}(z, t) & \text { if } r_{e}(z, t) \leq r \leq r_{c}(z, t)\end{cases}
$$


where $\bar{K}_{s}(z)$ and $\bar{K}_{c}(z, t)$ are the saturated conductivities of the soil and cortex, respectively, and $r_{c}(z, t)$ is the outer radius of the cortex. The boundary conditions for (2.8) are

$$
\begin{aligned}
& \bar{K}_{c}(z, t) \frac{\partial \psi_{r}}{\partial r}\left(z, r_{e}(z, t), t\right)=\left(2 \pi r_{e}(z, t) R(z, t)\right)^{-1}\left(\psi_{r}\left(z, r_{e}(z, t), t\right)-\psi_{x}(z, t)\right) \\
& \psi_{r}\left(z, r_{s}(z, t), t\right)=\psi_{s}(z, t)
\end{aligned}
$$

representing the resistive endodermal layer and continuity of potential, respectively, where $R(z, t)$ is the resistance of the endodermis per unit length of root. We should point out that there is a small inconsistency between the assumption of the subroot's soil cylinder being saturated and (2.10). A consistent approach would use $K_{s}$ in place of $\bar{K}_{s}$ in the definition of $K_{r}$; however, the current approach seems to be adequate in practice [15].

We assume that the amount of water extracted by the subroots at a given depth is directly proportional to the total linear length of subroots contained within the block at that depth. So let $\mathcal{L}(z, t)$ denote the root length density function. Finally, using (2.9), the coupling term is given by

$$
\mathcal{S}(z, t)=\mathcal{L}(z, t) A_{s} R^{-1}(z, t)\left(\psi_{r}\left(z, r_{e}(z, t), t\right)-\psi_{x}(z, t)\right) .
$$

Note that $(2.8)-(2.10)$ can be solved analytically since $K_{r}$ is a piece-wise constant function. Hence, with

$$
c(z, t)=\frac{1}{2 \pi}\left(\frac{1}{\bar{K}_{c}(z, t)} \ln \frac{r_{c}(z, t)}{r_{e}(z, t)}+\frac{1}{\bar{K}_{s}(z, t)} \ln \frac{r_{s}(z, t)}{r_{c}(z, t)}\right),
$$

we have the closed form solution

$$
\psi_{r}\left(z, r_{e}(z, t), t\right)=\frac{R(z, t)}{R(z, t)+c(z, t)} \psi_{s}(z, t)+\frac{c(z, t)}{R(z, t)+c(z, t)} \psi_{x}(z, t) .
$$

Now we can substitute this in (2.11) to obtain simply that

$$
\mathcal{S}(z, t)=S(z, t)\left(\psi_{s}(z, t)-\psi_{x}(z, t)\right) \quad \text { for } z \in I_{x}(t), t \in J
$$

where

$$
S(z, t)=\frac{\mathcal{L}(z, t) A_{s}}{R(z, t)+c(z, t)} .
$$

The full model is now (2.1)-(2.7) and (2.13). For further details and justifications of the assumptions we refer to [15] and [1].

We close this section with two remarks. First, without loss of generality, we assume that $L_{x}(t)=L$. If $L_{x}(t)<L$, then $\mathcal{L}(x, t)=0$ for $z>L_{x}(t)$, and the same solution is obtained since (2.5), (2.14), and (2.7) imply (2.7) with $L$ replacing $L_{x}(t)$. Second, for simplicity of exposition, we henceforth assume that the soil and taproot xylems are homogeneous and time independent, i.e., $K_{s}, A_{x}$, and $\bar{K}_{x}$ do not depend explicitly on $z$ or on $t$. With the obvious modifications, the rest of the paper carries through for the general case. 
3. A finite element scheme. In this section we define a finite element approximation scheme for our coupled system of equations. We begin by defining some notation and a weak form of the problem. Let $H^{k}$ denote the standard Sobolev space of $k$-differentiable functions in $L^{2}$. Let $(\cdot, \cdot)$ denote the $L^{2}(I)$-inner product,

$$
(u, v)=\int_{0}^{L} u v d z \quad \text { for any } u, v \in L^{2}(I)
$$

and let $\|\cdot\|_{k}$ denote the $H^{k}(I)$-norm. Then $\|u\|_{0}=(u, u)^{1 / 2}$ and, for integer $k$,

$$
\|u\|_{k}^{2}=\sum_{j=0}^{k}\left\|\frac{d^{j} u}{d z^{j}}\right\|_{0}^{2} .
$$

Also, set

$$
\|u\|_{L^{2}(J ; X)}^{2}=\int_{J}\|u(\cdot, t)\|_{X}^{2} d t \quad \text { and } \quad\|u\|_{L^{\infty}(J ; X)}=\underset{t \in J}{\operatorname{ess} \sup }\|u(\cdot, t)\|_{X} .
$$

Define the function spaces

$$
\mathcal{V}=\left\{v \in H^{1}(I) \mid v(L)=0\right\} \quad \text { and } \quad \mathcal{U}=H^{1}(I) .
$$

It is evident that if $\psi_{s}$ and $\psi_{x}$ constitute a solution to (2.1)-(2.7), (2.13), then $\psi_{s} \in$ $\mathcal{V}+\psi_{\text {bot }}$ and $\psi_{x} \in \mathcal{U}$ satisfies for any $t \in J$ the weak equations

$$
\begin{aligned}
& \left(\rho \frac{\partial \theta\left(\psi_{s}\right)}{\partial t}, v\right)+\left(K_{s}\left(\psi_{s}\right)\left(\frac{\partial \psi_{s}}{\partial z}-g\right), \frac{\partial v}{\partial z}\right) \\
& \quad=-\left[q_{\text {evap }}\left(\psi_{s}(0, \cdot)\right)-q_{\mathrm{prec}}\right] v(0)-\left(A_{s}^{-1} S\left(\psi_{s}-\psi_{x}\right), v\right) \text { for all } v \in \mathcal{V}
\end{aligned}
$$

and

$$
\begin{aligned}
& \left(\bar{K}_{x}\left(\frac{\partial \psi_{x}}{\partial z}-g\right), \frac{\partial u}{\partial z}\right) \\
& \quad=-A_{x}^{-1} q_{\text {trans }} u(0)+\left(A_{x}^{-1} S\left(\psi_{s}-\psi_{x}\right), u\right) \text { for all } u \in \mathcal{U} .
\end{aligned}
$$

Choose some partition $z_{0}=0<z_{1}<\cdots<z_{M}=L$ of $I$, and let $h_{i}=z_{i}-z_{i-1}$, $h=\max _{i} h_{i}$. Let $\mathcal{V}_{h}$ and $\mathcal{U}_{h}$ be standard finite element subspaces of $\mathcal{V}$ and $\mathcal{U}$ defined over the given partition of $I$ (see, e.g., [5]). Choose some partition $t_{0}=0<t_{1}<$ $\cdots<t_{N}=T$ of $J$, and let $\Delta t^{n}=t_{n}-t_{n-1}, \Delta t=\max _{n} \Delta t^{n}$. For any function $u(z, t)$, denote $u\left(z, t^{n}\right)$ by $u^{n}(z)$ and let the backward time difference operator be given by

$$
\partial_{t} u^{n}=\frac{u^{n}-u^{n-1}}{\Delta t^{n}}
$$

Our finite element scheme is the following: For $n=1, \ldots, N$, let $\Psi_{s}^{n} \in \mathcal{V}_{h}+\psi_{\text {bot }}^{n}$ and $\Psi_{x}^{n} \in \mathcal{U}_{h}$ satisfy

$$
\begin{aligned}
& \left(\rho \partial_{t} \theta\left(\Psi_{s}\right)^{n}, v\right)+\left(K_{s}\left(\Psi_{s}^{n}\right)\left(\frac{\partial \Psi_{s}^{n}}{\partial z}-g\right), \frac{\partial v}{\partial z}\right) \\
& \quad=-\left[q_{\text {evap }}^{n}\left(\Psi_{s}^{n}(0)\right)-q_{\mathrm{prec}}^{n}\right] v(0)-\left(A_{s}^{-1} S^{n}\left(\Psi_{s}^{n}-\Psi_{x}^{n}\right), v\right) \quad \text { for all } v \in \mathcal{V}_{h}
\end{aligned}
$$

and 


$$
\begin{aligned}
& \left(\bar{K}_{x}\left(\frac{\partial \Psi_{x}^{n}}{\partial z}-g\right), \frac{\partial u}{\partial z}\right) \\
& \quad=-A_{x}^{-1} q_{\mathrm{trans}}^{n} u(0)+\left(A_{x}^{-1} S^{n}\left(\Psi_{s}^{n}-\Psi_{x}^{n}\right), u\right) \text { for all } u \in \mathcal{U}_{h}
\end{aligned}
$$

where we initialize the procedure by choosing some reasonable approximation $\Psi_{s}^{0} \in$ $\mathcal{V}_{h}+\psi_{\text {bot }}^{0}$ to $\psi_{s}^{0}$. For example, one can take the interpolant of $\psi_{s}^{0}$ in $\mathcal{V}_{h}+\psi_{\text {bot }}^{0}$ or its elliptic projection (see (4.1) with $t=0$ below).

It can be shown that a unique solution exists for the scheme. An iterative procedure (such as Picard iteration or Newton's method [4]) can be used to compute the solution.

4. An error analysis for the finite element scheme. We make the following assumptions, wherein $c$ and $C$ are some fixed positive constants. (Recall that we have assumed that $L_{x}=L, K_{s}$ depends only on $\psi_{s}$, and $A_{x}$ and $\bar{K}_{x}$ are constants.)

(A1) $c \leq K_{s} \leq C,\left|K_{s}^{\prime}\right| \leq C,\left|K_{s}^{\prime \prime}\right| \leq C$, and $c \leq \bar{K}_{x} \leq C$.

(A2) $0 \leq S \leq C, c \leq S$ for $z$ in some nonempty interval for each $t \in \bar{J}$, and $|\partial S / \partial t| \leq C$.

(A3) There exists some fixed integer $p \geq 2$, such that for any $1 \leq q \leq p$,

$$
\begin{gathered}
\inf _{v \in \mathcal{V}_{h}}\|\psi-v\|_{1} \leq C\|\psi\|_{q} h^{q-1} \quad \text { for any } \psi \in \mathcal{V} \cap H^{q}(I), \\
\inf _{u \in \mathcal{U}_{h}}\|\psi-u\|_{1} \leq C\|\psi\|_{q} h^{q-1} \quad \text { for any } \psi \in \mathcal{U} \cap H^{q}(I) .
\end{gathered}
$$

(A4) $\psi_{s}, \partial \psi_{s} / \partial t$, and $\psi_{x} \in L^{2}\left(J ; H^{p}(I)\right)$.

(A5) $\partial \psi_{s} / \partial t \in L^{\infty}\left(J ; H^{2}(I)\right)$ and $\partial^{2} \psi_{s} / \partial t^{2} \in L^{2}\left(J ; L^{2}(I)\right)$.

(A6) $\left|q_{\text {evap }}\right| \leq C,\left|q_{\text {prec }}\right| \leq C,\left|q_{\text {trans }}\right| \leq C, 0 \leq \partial q_{\text {evap }} / \partial \psi \leq C,\left|\partial^{2} q_{\text {evap }} / \partial \psi^{2}\right| \leq C$, $\left|\partial q_{\mathrm{evap}} / \partial t\right| \leq C$, and $\left|\partial^{2} q_{\mathrm{evap}} / \partial \psi \partial t\right| \leq C$

(A7) $c \leq h_{i+1} / h_{i} \leq C$ and $\Delta t^{n+1} / \Delta t^{n} \leq C$.

(A8) $\psi_{\text {bot }} \leq 0$ and $\psi_{s}^{0} \in H^{p}(I)$. Moreover, $\Psi_{s}^{0}$ is defined so that $\left\|\Psi_{s}^{0}-\psi_{s}^{0}\right\|_{j} \leq$ $C\left\|\psi_{s}^{0}\right\|_{q} h^{q-j}, 1 \leq q \leq p, j=0,1$.

(A9) $c \leq \theta^{\prime} \leq C$ and $\left|\theta^{\prime \prime}\right| \leq C$ (physically, this essentially means that $-C \leq \psi_{s} \leq$ $-c)$.

Note that (A7) implies that the inverse inequality holds: For some fixed $C>0$,

$$
\|v\|_{L^{4}(I)} \leq C\|v\|_{0} h^{-1 / 4} \text { for any } v \in \mathcal{V}_{h} .
$$

Also, (A8) is easily satisfied by reasonable initialization procedures.

It should be noted that $\theta^{\prime}$ is in general a degenerate function: $\theta^{\prime}\left(\psi_{s}\right)=0$ precisely when $\psi_{s} \geq 0$ (the saturated region) and $\theta^{\prime}\left(\psi_{s}\right) \rightarrow 0$ as $\psi_{s} \rightarrow-\infty$ (the dry region) [10]. Since most plants die in cases of either extreme, we have restricted ourselves to the case defined by (A9) above.

THEOREM 1. Assume (A1)-(A9). Then for $h$ and $\Delta t$ sufficiently small and for any $1 \leq q \leq p$,

(i) $\max _{n}\left\|\Psi_{s}^{n}-\psi_{s}^{n}\right\|_{0} \leq C\left\{\left[\left(\sum_{n=1}^{N}\left(\left\|\frac{\partial \psi_{s}^{n}}{\partial t}\right\|_{q}^{2}+\left\|\psi_{x}^{n}\right\|_{q}^{2}\right) \Delta t^{n}\right)^{1 / 2}\right.\right.$

$$
\left.\left.+\left\|\psi_{s}\right\|_{L^{\infty}\left(J ; H^{q}(I)\right)}+\left\|\psi_{s}^{0}\right\|_{q}\right] h^{q}+\Delta t\right\}
$$

(ii) $\max _{n}\left\|\Psi_{x}^{n}-\psi_{x}^{n}\right\|_{0} \leq C\left\{\left[\left(\sum_{n=1}^{N}\left\|\frac{\partial \psi_{s}^{n}}{\partial t}\right\|_{q}^{2} \Delta t^{n}\right)^{1 / 2}+\left\|\psi_{s}\right\|_{L^{\infty}\left(J ; H^{q}(I)\right)}\right.\right.$

$$
\left.\left.+\left\|\psi_{x}\right\|_{L^{\infty}\left(J ; H^{q}(I)\right)}+\left\|\psi_{s}^{0}\right\|_{q}\right] h^{q}+\Delta t\right\},
$$


(iii) $\max _{n}\left\|\frac{\partial}{\partial z}\left(\Psi_{s}^{n}-\psi_{s}^{n}\right)\right\|_{0} \leq C\left\{\left[\left(\sum_{n=1}^{N}\left\|\frac{\partial \psi_{s}^{n}}{\partial t}\right\|_{q-1}^{2} \Delta t^{n}\right)^{1 / 2}+\left\|\psi_{s}\right\|_{L^{\infty}\left(J ; H^{q}(I)\right)}\right.\right.$ $\left.\left.+\left\|\psi_{x}\right\|_{L^{\infty}\left(J ; H^{q-1}(I)\right)}+\left\|\psi_{s}^{0}\right\|_{q}\right] h^{q-1}+\Delta t\right\}$

(iv) $\max _{n}\left\|\frac{\partial}{\partial z}\left(\Psi_{x}^{n}-\psi_{x}^{n}\right)\right\|_{0} \leq C\left\{\left[\left(\sum_{n=1}^{N}\left\|\frac{\partial \psi_{s}^{n}}{\partial t}\right\|_{q-1}^{2} \Delta t^{n}\right)^{1 / 2}+\left\|\psi_{s}\right\|_{L^{\infty}\left(J ; H^{q-1}(I)\right)}\right.\right.$ $\left.\left.+\left\|\psi_{x}\right\|_{L^{\infty}\left(J ; H^{q}(I)\right)}+\left\|\psi_{s}^{0}\right\|_{q-1}\right] h^{q-1}+\Delta t\right\}$,

(v) $\left(\sum_{n=1}^{N}\left\|\partial_{t}\left(\Psi_{s}-\psi_{s}\right)^{n}\right\|_{0}^{2} \Delta t^{n}\right)^{1 / 2} \leq C\left\{\left[\left(\sum_{n=1}^{N}\left\|\frac{\partial \psi_{s}^{n}}{\partial t}\right\|_{q-1}^{2} \Delta t^{n}\right)^{1 / 2}\right.\right.$

$$
\left.\left.+\left\|\psi_{s}\right\|_{L^{\infty}\left(J ; H^{q-1}(I)\right)}+\left\|\psi_{x}\right\|_{L^{\infty}\left(J ; H^{q-1}(I)\right)}+\left\|\psi_{s}^{0}\right\|_{q}\right] h^{q-1}+\Delta t\right\}
$$

where $C$ is independent of $h$ and $\Delta t$.

We will derive error estimates by using the technique of comparing the approximate solution to an elliptic projection of the exact solution [16], which we now define. For $t \in J$, let $\tilde{\Psi}_{s}(\cdot, t) \in \mathcal{V}_{h}+\psi_{\text {bot }}(t)$ satisfy

$$
\left(K_{s}\left(\psi_{s}\right) \frac{\partial}{\partial z}\left(\psi_{s}-\tilde{\Psi}_{s}\right), \frac{\partial v}{\partial z}\right)+\left(A_{s}^{-1} S\left(\psi_{s}-\tilde{\Psi}_{s}\right), v\right)=0 \text { for all } v \in \mathcal{V}_{h}
$$

and let $\tilde{\Psi}_{x}(z, t) \in \mathcal{U}_{h}$ satisfy

$$
\left(\bar{K}_{x} \frac{\partial}{\partial z}\left(\psi_{x}-\tilde{\Psi}_{x}\right), \frac{\partial u}{\partial z}\right)+\left(A_{x}^{-1} S\left(\psi_{x}-\tilde{\Psi}_{x}\right), u\right)=0 \quad \text { for all } u \in \mathcal{U}_{h} .
$$

Theorem 2. Assume (A1)-(A9). Then at a.e. time $t$, for any $z_{i}, n \geq 0$, and $1 \leq q \leq p$,

(i) $\left\|\psi_{s}-\tilde{\Psi}_{s}\right\|_{0}+\left\|\psi_{s}-\tilde{\Psi}_{s}\right\|_{1} h \leq C\left\|\psi_{s}\right\|_{q} h^{q}$,

(ii) $\left\|\frac{\partial \tilde{\Psi}_{s}}{\partial z}\right\|_{L^{\infty}(I)} \leq C\left\|\psi_{s}\right\|_{2}$

(iii) $\left|\psi_{s}\left(z_{i}, \cdot\right)-\tilde{\Psi}_{s}\left(z_{i}, \cdot\right)\right| \leq C\left\|\psi_{s}\right\|_{q} h^{q}$,

(iv) $\left\|\frac{\partial}{\partial t}\left(\psi_{s}-\tilde{\Psi}_{s}\right)\right\|_{0} \leq C\left\{\left\|\psi_{s}\right\|_{q}+\left\|\frac{\partial \psi_{s}}{\partial t}\right\|_{q}\right\} h^{q}$,

(v) $\left\|\frac{\partial^{2} \tilde{\Psi}_{s}}{\partial z \partial t}\right\|_{L^{\infty}(I)} \leq C\left\{\left\|\psi_{s}\right\|_{2}+\left\|\frac{\partial \psi_{s}}{\partial t}\right\|_{2}\right\}$,

(vi) $\left|\frac{\partial}{\partial t}\left(\psi_{s}\left(z_{i}\right)-\tilde{\Psi}_{s}\left(z_{i}\right)\right)\right| \leq C\left\{\left\|\psi_{s}\right\|_{q}+\left\|\frac{\partial \psi_{s}}{\partial t}\right\|_{q}\right\}^{q}$,

(vii) $\left\|\psi_{x}-\tilde{\Psi}_{x}\right\|_{0}+\left\|\psi_{x}-\tilde{\Psi}_{x}\right\|_{1} h \leq C\left\|\psi_{x}\right\|_{q} h^{q}$, where $C$ is independent of $h, t, z_{i}$, and $n$.

Proof. Most of these error estimates are well known from elliptic approximation theory [5], [9], [16]. Results (iii) and (vi) hold since in one dimension we have superconvergence at the points of the spatial partition [7].

We remark that we can weaken (ii) by replacing the $H^{2}$-norm on the right side by the $H^{3 / 2}$-norm. A similar statement applies to (v). Also, we can improve (iii) and 
(vi) by replacing $h^{q}$ by $h^{q+p-2}$ if $\mathcal{V}_{h}$ is the space of continuous, piecewise polynomials of degree $p-1$ over the partition.

We shall also need two results on the approximation of nonlinear accumulation terms. The first result is due to Arbogast [2], whereas the second is a modification of it. We note in passing that the well known inequality

$$
a b \leq \epsilon a^{2}+\frac{1}{4 \epsilon} b^{2}
$$

will be used often below without further comment; it holds for $\epsilon>0$ and $a, b \in \mathbb{R}$. Also, $Q$ will denote a generic positive constant that is independent of $h, n$, and the $z_{i}$ and $\Delta t^{n}$.

LEMMA 1. Suppose that $u^{n}, u^{n-1}, v^{n}$, and $v^{n-1}$ are real numbers. Suppose also that $\gamma: \mathbb{R} \rightarrow \mathbb{R}$ is such that $0 \leq \gamma^{\prime} \leq C<\infty$ and $\left|\gamma^{\prime \prime}\right| \leq C$. Then

where

$$
\partial_{t}(\gamma(u)-\gamma(v))^{n}\left(u^{n}-v^{n}\right)=\partial_{t}\left(\int_{v}^{u}[\gamma(\mu)-\gamma(v)] d \mu\right)^{n}-E,
$$

$$
E \leq C^{\prime}\left\{\left(u^{n}-v^{n}\right)^{2}+\left(u^{n-1}-v^{n-1}\right)^{2}+\left(\Delta t^{n}\right)^{2}\right\}
$$

for some $C^{\prime}$ depending on $\left|\partial_{t} u^{n}\right|$ and $C$.

Proof. The proof appears in [2]; we reproduce it here for completeness. We exploit the fact that $\left|u^{n}-u^{n-1}\right|$ can be replaced by $\left|\partial_{t} u^{n}\right| \Delta t^{n} \leq Q \Delta t^{n}$.

First note that in fact

$$
\begin{aligned}
E=\left(\gamma\left(u^{n}\right)-\gamma\left(v^{n}\right)\right) \partial_{t} u^{n}-\partial_{t} \gamma(u)^{n}\left(u^{n}-v^{n}\right)+\left(\gamma\left(v^{n}\right)-\gamma\left(v^{n-1}\right)\right) \partial_{t} u^{n} \\
-\frac{1}{\Delta t^{n}} \int_{u^{n-1}}^{u^{n}}\left(\gamma\left(u^{n}\right)-\gamma(\mu)\right) d \mu-\frac{1}{\Delta t^{n}} \int_{v^{n-1}}^{v^{n}}\left(\gamma(\mu)-\gamma\left(v^{n-1}\right)\right) d \mu .
\end{aligned}
$$

For the first two terms above, there are $\nu_{1}$ (between $u^{n}$ and $v^{n}$ ) and $\nu_{2}$ (between $u^{n}$ and $u^{n-1}$ ) such that

$$
\begin{aligned}
&\left(\gamma\left(u^{n}\right)-\gamma\left(v^{n}\right)\right) \partial_{t} u^{n}-\partial_{t} \gamma(u)^{n}\left(u^{n}-v^{n}\right)=\left(\gamma^{\prime}\left(\nu_{1}\right)-\gamma^{\prime}\left(\nu_{2}\right)\right)\left(u^{n}-v^{n}\right) \partial_{t} u^{n} \\
& \leq Q\left|\left(\nu_{1}-\nu_{2}\right)\left(u^{n}-v^{n}\right)\right| \leq Q\left\{\left(u^{n}-v^{n}\right)^{2}+\left(\Delta t^{n}\right)^{2}\right\}
\end{aligned}
$$

where $Q$ depends the bound for $\left|\gamma^{\prime \prime}\right|$ and on $\left|\partial_{t} u^{n}\right|$. For the first integral term in (4.3), note that for some $\nu_{3}$ and $\nu_{4}$ between $u^{n}$ and $u^{n-1}$,

$$
\int_{u^{n-1}}^{u^{n}}\left(\gamma\left(u^{n}\right)-\gamma(\mu)\right) d \mu=\int_{u^{n-1}}^{u^{n}} \gamma^{\prime}\left(\nu_{3}(\mu)\right)\left(u^{n}-\mu\right) d \mu=\frac{1}{2} \gamma^{\prime}\left(\nu_{4}\right)\left(u^{n}-u^{n-1}\right)^{2} .
$$

A similar expression holds for the other integral with some $\nu_{5}$ between $v^{n}$ and $v^{n-1}$. Finally, for some $\nu_{6}$ between $v^{n}$ and $v^{n-1}$, the last three terms of (4.3) are

$$
\begin{aligned}
\left(2 \Delta t^{n}\right)^{-1}\left\{2 \gamma^{\prime}\left(\nu_{6}\right)\left(v^{n}-v^{n-1}\right)\left(u^{n}-u^{n-1}\right)\right. & \\
& \left.-\gamma^{\prime}\left(\nu_{4}\right)\left(u^{n}-u^{n-1}\right)^{2}-\gamma^{\prime}\left(\nu_{5}\right)\left(v^{n}-v^{n-1}\right)^{2}\right\} \\
= & \left(2 \Delta t^{n}\right)^{-1}\left\{2\left[\gamma^{\prime}\left(\nu_{6}\right)-\gamma^{\prime}\left(\nu_{5}\right)\right]\left(v^{n}-v^{n-1}\right)\left(u^{n}-u^{n-1}\right)\right. \\
& +2 \gamma^{\prime}\left(\nu_{5}\right)\left(v^{n}-v^{n-1}\right)\left(u^{n}-u^{n-1}\right) \\
& \left.-\gamma^{\prime}\left(\nu_{4}\right)\left(u^{n}-u^{n-1}\right)^{2}-\gamma^{\prime}\left(\nu_{5}\right)\left(v^{n}-v^{n-1}\right)^{2}\right\} \\
\leq & \left(2 \Delta t^{n}\right)^{-1}\left\{2\left[\gamma^{\prime}\left(\nu_{6}\right)-\gamma^{\prime}\left(\nu_{5}\right)\right]\left(v^{n}-v^{n-1}\right)\left(u^{n}-u^{n-1}\right)\right. \\
& \left.+\left[\gamma^{\prime}\left(\nu_{5}\right)-\gamma^{\prime}\left(\nu_{4}\right)\right]\left(u^{n}-u^{n-1}\right)^{2}\right\} \\
\leq & Q\left\{\left|\nu_{6}-\nu_{5}\right|\left|v^{n}-v^{n-1}\right|+\left|\nu_{5}-\nu_{4}\right| \Delta t^{n}\right\} \\
\leq & Q\left\{\left(u^{n}-v^{n}\right)^{2}+\left(u^{n-1}-v^{n-1}\right)^{2}+\left(\Delta t^{n}\right)^{2}\right\},
\end{aligned}
$$


where $Q$ depends on the bound for $\left|\gamma^{\prime \prime}\right|$ and on $\left|\partial_{t} u^{n}\right|$. Combining (4.3)-(4.6), we have the lemma. [

LeMmA 2. Suppose that $u^{n}, u^{n-1}, u^{n}$, and $v^{n-1}$ are real numbers. Suppose also that $\gamma(t, u): \mathbb{R} \times \mathbb{R} \rightarrow \mathbb{R}$ is such that $0 \leq \partial \gamma / \partial u \leq C<\infty,\left|\partial^{2} \gamma / \partial u^{2}\right| \leq C$, $|\partial \gamma / \partial t| \leq C$, and $\left|\partial^{2} \gamma / \partial u \partial t\right| \leq C$. Then

$$
\left(\gamma^{n}\left(u^{n}\right)-\gamma^{n}\left(v^{n}\right)\right) \partial_{t}(u-v)^{n}=\partial_{t}\left(\int_{v}^{u}[\gamma(u)-\gamma(\mu)] d \mu\right)^{n}-E
$$

where

$$
E \leq C^{\prime}\left\{\left(u^{n}-v^{n}\right)^{2}+\left(u^{n-1}-v^{n-1}\right)^{2}+\left(\Delta t^{n}\right)^{2}\right\}
$$

for some $C^{\prime}$ depending on $\left|\partial_{t} u^{n}\right|$ and $C$.

Proof. We write $E$ as follows:

$$
\begin{aligned}
E= & \left(\Delta t^{n}\right)^{-1}\left[\gamma^{n}\left(u^{n}\right)-\gamma^{n}\left(u^{n-1}\right)\right]\left(u^{n}-v^{n}\right)-\left(\gamma^{n}\left(u^{n}\right)-\gamma^{n}\left(v^{n}\right)\right) \partial_{t} u^{n} \\
& +\left(\gamma^{n}\left(u^{n}\right)-\gamma^{n}\left(u^{n-1}\right)\right) \partial_{t} v^{n} \\
& -\frac{1}{\Delta t^{n}} \int_{u^{n-1}}^{u^{n}}\left(\gamma^{n}(\mu)-\gamma^{n}\left(u^{n-1}\right)\right) d \mu-\frac{1}{\Delta t^{n}} \int_{v^{n-1}}^{v^{n}}\left(\gamma^{n}\left(v^{n}\right)-\gamma^{n}(\mu)\right) d \mu \\
& +\left(\Delta t^{n}\right)^{-1}\left[\gamma^{n}\left(u^{n-1}\right)-\gamma^{n-1}\left(u^{n-1}\right)\right]\left(u^{n-1}-v^{n-1}\right) \\
& -\frac{1}{\Delta t^{n}} \int_{v^{n-1}}^{u^{n-1}}\left(\gamma^{n}(\mu)-\gamma^{n-1}(\mu)\right) d \mu .
\end{aligned}
$$

The first five terms above are handled as in the proof of Lemma 1, where the bounding constant will depend on the bound for $\left|\partial^{2} \gamma^{n} / \partial u^{2}\right|$ and on $\left|\partial_{t} u^{n}\right|$.

For the final two terms in (4.7), we estimate as follows:

$$
\begin{aligned}
\left(\Delta t^{n}\right)^{-1}\left[\gamma^{n}\left(u^{n-1}\right)-\gamma^{n-1}\left(u^{n-1}\right)\right]\left(u^{n-1}-v^{n-1}\right) \\
\quad-\frac{1}{\Delta t^{n}} \int_{v^{n-1}}^{u^{n-1}}\left(\gamma^{n}(\mu)-\gamma^{n-1}(\mu)\right) d \mu \\
=\frac{1}{\Delta t^{n}} \int_{t_{n-1}}^{t_{n}} \int_{v^{n-1}}^{u^{n-1}}\left(\frac{\partial \gamma\left(\tau, u^{n-1}\right)}{\partial t}-\frac{\partial \gamma(\tau, \mu)}{\partial t}\right) d \mu d \tau \\
\leq Q\left(u^{n-1}-v^{n-1}\right)^{2}
\end{aligned}
$$

for some $Q$ depending on the bound for $\left|\partial^{2} \gamma / \partial t \partial u\right|$. $\square$

Proof of Theorem 1. Let

$$
\begin{array}{ll}
\xi^{n}=\Psi_{s}^{n}-\tilde{\Psi}_{s}^{n}, & \eta^{n}=\Psi_{x}^{n}-\tilde{\Psi}_{x}^{n} \\
\alpha^{n}=\tilde{\Psi}_{s}^{n}-\psi_{s}^{n}, & \beta^{n}=\tilde{\Psi}_{x}^{n}-\psi_{x}^{n} .
\end{array}
$$

Substituting (4.1) in (3.1) and subtracting the result from (3.3), we have that

$$
\begin{aligned}
\left(\rho\left[\partial_{t} \theta\left(\Psi_{s}\right)^{n}-\partial_{t} \theta\left(\psi_{s}\right)^{n}\right], v\right) \\
\quad+\left(K_{s}\left(\Psi_{s}^{n}\right)\left(\frac{\partial \Psi_{s}^{n}}{\partial z}-g\right)-K_{s}\left(\psi_{s}^{n}\right)\left(\frac{\partial \tilde{\Psi}_{s}^{n}}{\partial z}-g\right), \frac{\partial v}{\partial z}\right) \\
+\left[q_{\text {evap }}^{n}\left(\Psi_{s}^{n}(0)\right)-q_{\text {evap }}^{n}\left(\psi_{s}^{n}(0)\right)\right] v(0) \\
=-\left(A_{s}^{-1} S^{n}\left[\xi^{n}-\left(\eta^{n}+\beta^{n}\right)\right], v\right) \\
\quad-\left(\rho\left[\partial_{t} \theta\left(\psi_{s}\right)^{n}-\frac{\partial \theta\left(\psi_{s}^{n}\right)}{\partial t}\right], v\right) \text { for all } v \in \mathcal{V}_{h} .
\end{aligned}
$$


Similarly, from (4.2), (3.2), and (3.4) we have that

$$
\begin{array}{r}
\left(\bar{K}_{x} \frac{\partial \eta^{n}}{\partial z}, \frac{\partial u}{\partial z}\right)+\left(A_{x}^{-1} S^{n} \eta^{n}, u\right)=\left(A_{x}^{-1} S^{n}\left(\xi^{n}+\alpha^{n}\right), u\right) \\
\text { for all } u \in \mathcal{U}_{h} .
\end{array}
$$

Equation (4.10) with $u=\eta^{n} \in \mathcal{U}_{h}$ is easily estimated; by Poincaré's inequality (which holds since at all times $S \not \equiv 0$ ),

$$
\left\|\eta^{n}\right\|_{1}^{2} \leq Q\left(\left\|\xi^{n}\right\|_{0}^{2}+\left\|\alpha^{n}\right\|_{0}^{2}\right)
$$

where $Q$ depends on $A_{x}$ and on the bounds for $S$ and $\bar{K}_{x}$ (recall that $Q$ is a generic positive constant).

Let $v=\xi^{n} \in \mathcal{V}_{h}$ in (4.9). For some $\nu^{n}$ between $\Psi_{s}^{n}$ and $\psi_{s}^{n}$, we obtain after some manipulation that

$$
\begin{aligned}
\left(\rho\left[\partial_{t} \theta\left(\Psi_{s}\right)^{n}-\partial_{t} \theta\left(\psi_{s}\right)^{n}\right], \Psi_{s}^{n}-\psi_{s}^{n}\right)+\left(K_{s}\left(\Psi_{s}^{n}\right) \frac{\partial \xi^{n}}{\partial z}, \frac{\partial \xi^{n}}{\partial z}\right) \\
+\left[q_{\text {evap }}^{n}\left(\Psi_{s}^{n}(0)\right)-q_{\text {evap }}^{n}\left(\psi_{s}^{n}(0)\right)\right]\left(\Psi_{s}^{n}(0)-\psi_{s}^{n}(0)\right)+\left(A_{s}^{-1} S^{n} \xi^{n}, \xi^{n}\right) \\
=\left[q_{\text {evap }}^{n}\left(\Psi_{s}^{n}(0)\right)-q_{\text {evap }}^{n}\left(\psi_{s}^{n}(0)\right)\right] \alpha^{n}(0)+\left(A_{s}^{-1} S^{n}\left(\eta^{n}+\beta^{n}\right), \xi^{n}\right) \\
-\left(K_{s}^{\prime}\left(\nu^{n}\right)\left(\xi^{n}+\alpha^{n}\right)\left(\frac{\partial \tilde{\Psi}_{s}^{n}}{\partial z}-g\right), \frac{\partial \xi^{n}}{\partial z}\right) \\
-\left(\rho\left[\partial_{t} \theta\left(\psi_{s}\right)^{n}-\frac{\partial \theta\left(\psi_{s}^{n}\right)}{\partial t}\right], \xi^{n}\right)+\left(\rho\left[\partial_{t} \theta\left(\Psi_{s}\right)^{n}-\partial_{t} \theta\left(\psi_{s}\right)^{n}\right], \alpha^{n}\right) .
\end{aligned}
$$

For the first term on the left side, we invoke Lemma 1:

$$
\begin{aligned}
\left(\partial_{t} \theta\left(\Psi_{s}\right)^{n}-\partial_{t} \theta\left(\psi_{s}\right)^{n}\right)\left(\Psi_{s}^{n}-\psi_{s}^{n}\right) & \\
\geq \partial_{t} & \left(\int_{\Psi_{s}}^{\psi_{s}}\left(\theta(\mu)-\theta\left(\Psi_{s}\right)\right) d \mu\right)^{n} \\
& \quad-Q\left\{\left(\xi^{n}\right)^{2}+\left(\xi^{n-1}\right)^{2}+\left(\alpha^{n}\right)^{2}+\left(\alpha^{n-1}\right)^{2}+\left(\Delta t^{n}\right)^{2}\right\},
\end{aligned}
$$

where $Q$ depends on the upper bound of $\left|\theta^{\prime \prime}\right|$ and on $\left\|\partial \psi_{s} / \partial t\right\|_{L^{\infty}\left(J ; L^{\infty}(I)\right)}$. Easily

$$
\begin{aligned}
\left\|\partial_{t} \theta\left(\psi_{s}\right)^{n}-\frac{\partial \theta\left(\psi_{s}^{n}\right)}{\partial t}\right\|_{0} & =\left\|\frac{1}{\Delta t^{n}} \int_{t_{n-1}}^{t_{n}} \frac{\partial^{2} \theta\left(\psi_{s}^{n}\right)}{\partial t^{2}}\left(t-t_{n-1}\right) d t\right\|_{0} \\
& \leq\left(\frac{\Delta t^{n}}{3}\right)^{1 / 2}\left\|\frac{\partial^{2} \theta\left(\psi_{s}^{n}\right)}{\partial t^{2}}\right\|_{L^{2}\left(\left(t_{n-1}, t_{n}\right) ; L^{2}(I)\right)} .
\end{aligned}
$$

Hence, combining (4.12)--(4.14), we obtain with (4.11) that

$$
\begin{aligned}
\int_{0}^{L} \rho \partial_{t}\left(\int_{\Psi_{s}}^{\psi_{s}}\left(\theta(\mu)-\theta\left(\Psi_{s}\right)\right) d \mu\right)^{n} d z+\frac{1}{2}\left(K_{s}\left(\Psi_{s}^{n}\right) \frac{\partial \xi^{n}}{\partial z}, \frac{\partial \xi^{n}}{\partial z}\right) \\
+\frac{1}{2}\left[q_{\mathrm{evap}}^{n}\left(\Psi_{s}^{n}(0)\right)-q_{\mathrm{evap}}^{n}\left(\psi_{s}^{n}(0)\right)\right]\left(\Psi_{s}^{n}(0)-\psi_{s}^{n}(0)\right)+\left(A_{s}^{-1} S^{n} \xi^{n}, \xi^{n}\right) \\
\leq Q\left\{\left\|\xi^{n}\right\|_{0}^{2}+\left\|\xi^{n-1}\right\|_{0}^{2}+\left\|\alpha^{n}\right\|_{0}^{2}+\left\|\alpha^{n-1}\right\|_{0}^{2}+\left(\alpha^{n}(0)\right)^{2}+\left\|\beta^{n}\right\|_{0}^{2}\right. \\
\left.+\left(\Delta t^{n}\right)^{2}+\Delta t^{n}\left\|\frac{\partial^{2} \theta\left(\psi_{s}^{n}\right)}{\partial t^{2}}\right\|_{L^{2}\left(\left(t_{n-1}, t_{n}\right) ; L^{2}(I)\right)}^{2}\right\} \\
+\left(\rho\left[\partial_{t} \theta\left(\Psi_{s}\right)^{n}-\partial_{t} \theta\left(\psi_{s}\right)^{n}\right], \alpha^{n}\right),
\end{aligned}
$$


where $Q$ depends on $A_{x}, A_{s},\left\|\partial \tilde{\Psi}_{s} / \partial t\right\|_{L^{\infty}\left(J_{;} L^{\infty}(I)\right)},\left\|\partial \psi_{s} / \partial t\right\|_{L^{\infty}\left(J ; L^{\infty}(I)\right)}, g, \rho$, and on various bounds for $\bar{K}_{x}, K_{s}, K_{s}^{\prime}, S, \theta^{\prime \prime}$, and $q_{\text {evap }}$.

Multiplying (4.15) through by $\Delta t^{n}$ and summing on $n$ from 1 to $\bar{n} \leq N$, the first term on the left collapses while the last term on the right can be summed by parts:

$$
\begin{aligned}
\sum_{n=1}^{\bar{n}}\left(\rho\left[\partial_{t} \theta\left(\Psi_{s}\right)^{n}-\partial_{t} \theta\left(\psi_{s}\right)^{n}\right], \alpha^{n}\right) \Delta t^{n} & \left(\rho\left[\theta\left(\Psi_{s}^{\bar{n}}\right)-\theta\left(\psi_{s}^{\bar{n}}\right)\right], \alpha^{\bar{n}}\right)-\left(\rho\left[\theta\left(\Psi_{s}^{0}\right)-\theta\left(\psi_{s}^{0}\right)\right], \alpha^{1}\right) \\
= & \quad-\sum_{n=1}^{\bar{n}-1}\left(\rho\left[\theta\left(\Psi_{s}\right)^{n}-\theta\left(\psi_{s}\right)^{n}\right], \partial_{t} \alpha^{n+1}\right) \frac{\Delta t^{n+1}}{\Delta t^{n}} \Delta t^{n}
\end{aligned}
$$

Since in general

$$
Q^{-1}\|v-u\|_{0}^{2} \leq \int_{u}^{v}(\theta(\mu)-\theta(u)) d \mu \leq Q\|v-u\|_{0}^{2}
$$

(wherein $Q$ depends on the upper and lower bounds for $\theta^{\prime}$ ), after some manipulation we obtain from (4.15) that

$$
\begin{aligned}
\| \Psi_{s}^{\bar{n}} & -\psi_{s}^{\bar{n}}\left\|_{0}^{2}+\sum_{n=1}^{\bar{n}}\right\| \frac{\partial \xi^{n}}{\partial z} \|_{0}^{2} \Delta t^{n} \\
& +\sum_{n=1}^{\bar{n}}\left[q_{\text {evap }}^{n}\left(\Psi_{s}^{n}(0)\right)-q_{\text {evap }}^{n}\left(\psi_{s}^{n}(0)\right)\right]\left(\Psi_{s}^{n}(0)-\psi_{s}^{n}(0)\right) \Delta t^{n} \\
\leq & Q\left\{\sum_{n=1}^{\bar{n}}\left[\left\|\Psi_{s}^{n}-\psi_{s}^{n}\right\|_{0}^{2}+\left\|\frac{\partial \alpha^{n}}{\partial t}\right\|_{0}^{2}+\left(\alpha^{n}(0)\right)^{2}+\left\|\beta^{n}\right\|_{0}^{2}\right] \Delta t^{n}\right. \\
& \left.+\|\alpha\|_{L^{\infty}\left(J ; L^{2}(I)\right)}^{2}+\left\|\Psi_{s}^{0}-\psi_{s}^{0}\right\|_{0}^{2}+(\Delta t)^{2}\right\},
\end{aligned}
$$

where $Q$ depends on all the previous factors and on $T$ and on bounds for $\Delta t^{n+1} / \Delta t^{n}$, $\partial^{2} \psi_{s} / \partial t^{2}, \theta^{\prime}$, and $\theta^{\prime \prime}$. Finally, for a small enough $\Delta t$, Gronwall's Theorem allows us to remove the first term on the right side above.

With Theorem 2, (4.17) gives part (i) of Theorem 1. Parts (ii) and (iv) are also now obtained from part (i), (4.11), and Theorem 2 .

To obtain the remaining parts of Theorem 1 , let us now take $v=\partial_{t} \xi^{n} \in \mathcal{V}_{h}$ in (4.9). Since

$$
2 \frac{\partial \xi^{n}}{\partial z} \partial_{t}\left(\frac{\partial \xi}{\partial z}\right)^{n}=\partial_{t}\left(\left[\frac{\partial \xi}{\partial z}\right]^{2}\right)^{n}+\left[\partial_{t}\left(\frac{\partial \xi}{\partial z}\right)^{n}\right]^{2} \Delta t^{n}
$$


after some manipulation we see that

$$
\begin{aligned}
\left(\rho \left[\partial_{t}\right.\right. & \left.\left.\theta\left(\Psi_{s}\right)^{n}-\partial_{t} \theta\left(\psi_{s}\right)^{n}\right], \partial_{t} \xi^{n}\right) \\
& +\frac{1}{2}\left(K_{s}\left(\Psi_{s}^{n}\right), \partial_{t}\left(\left[\frac{\partial \xi}{\partial z}\right]^{2}\right)^{n}+\left[\partial_{t}\left(\frac{\partial \xi}{\partial z}\right)^{n}\right]^{2} \Delta t^{n}\right) \\
& +\left[q_{\text {evap }}^{n}\left(\Psi_{s}^{n}(0)\right)-q_{\text {evap }}^{n}\left(\psi_{s}^{n}(0)\right)\right] \partial_{t} \xi^{n}(0) \\
= & \left(\left[K_{s}\left(\psi_{s}^{n}\right)-K_{s}\left(\Psi_{s}^{n}\right)\right]\left(\frac{\partial \tilde{\Psi}_{s}^{n}}{\partial z}-g\right), \partial_{t}\left(\frac{\partial \xi}{\partial z}\right)^{n}\right) \\
& -\left(A_{s}^{-1} S^{n}\left[\xi^{n}-\left(\eta^{n}+\beta^{n}\right)\right], \partial_{t} \xi^{n}\right) \\
& -\left(\rho\left[\partial_{t} \theta\left(\psi_{s}\right)^{n}-\frac{\partial \theta\left(\psi_{s}^{n}\right)}{\partial t}\right], \partial_{t} \xi^{n}\right) .
\end{aligned}
$$

For the first term, note that for some $\nu_{1}^{n}$ between $\Psi_{s}^{n}$ and $\Psi_{s}^{n-1}$ and some $\nu_{2}^{n}$ between $\psi_{s}^{n}$ and $\psi_{s}^{n-1}$,

$$
\begin{aligned}
& \left(\rho\left[\partial_{t} \theta\left(\Psi_{s}\right)^{n}-\partial_{t} \theta\left(\psi_{s}\right)^{n}\right], \partial_{t} \xi^{n}\right) \\
& \quad=\left(\rho\left[\theta^{\prime}\left(\nu_{1}^{n}\right) \partial_{t} \Psi_{s}^{n}-\theta^{\prime}\left(\nu_{2}^{n}\right) \partial_{t} \psi_{s}^{n}\right], \partial_{t} \xi^{n}\right) \\
& =\left(\rho \theta^{\prime}\left(\nu_{1}^{n}\right) \partial_{t}(\xi+\alpha)^{n}, \partial_{t} \xi^{n}\right)+\left(\rho\left[\theta^{\prime}\left(\nu_{1}^{n}\right)-\theta^{\prime}\left(\nu_{2}^{n}\right)\right] \partial_{t} \psi_{s}^{n}, \partial_{t} \xi^{n}\right) \\
& \geq q\left\|\partial_{t} \xi^{n}\right\|_{0}^{2}-Q\left\{\left\|\nu_{1}^{n}-\nu_{2}^{n}\right\|_{0}^{2}+\left\|\partial_{t} \alpha^{n}\right\|_{0}^{2}\right\} \\
& \geq q\left\|\partial_{t} \xi^{n}\right\|_{0}^{2}-Q\left\{\left\|\xi^{n}\right\|_{0}^{2}+\left\|\xi^{n-1}\right\|_{0}^{2}+\left\|\alpha^{n}\right\|_{0}^{2}+\left\|\alpha^{n-1}\right\|_{0}^{2}\right. \\
& \left.+\left\|\partial_{t} \alpha^{n}\right\|_{0}^{2}+\left(\Delta t^{n}\right)^{2}\right\}
\end{aligned}
$$

where $q>0$ and $Q$ depend on $\rho$, the lower bound for $\theta^{\prime}$, the upper bound for $\left|\theta^{\prime \prime}\right|$, and on $\left\|\partial \psi_{s} / \partial t\right\|_{L^{\infty}\left(J ; L^{\infty}(I)\right)}$.

For two of the other terms in (4.19), we should first note that for some $\nu_{3}^{n}$ between $\psi_{s}^{n}$ and $\psi_{s}^{n-1}$ and $\nu_{4}^{n}$ between $\Psi_{s}^{n}$ and $\Psi_{s}^{n-1}$

$$
\begin{aligned}
& \left|\partial_{t}\left(K_{s}\left(\psi_{s}\right)-K_{s}\left(\Psi_{s}\right)\right)^{n}\right| \\
& \quad=\left|K_{s}^{\prime}\left(\nu_{3}^{n}\right) \partial_{t} \psi_{s}^{n}-K_{s}^{\prime}\left(\nu_{4}^{n}\right) \partial_{t} \Psi_{s}^{n}\right| \\
& \quad=\left|-K_{s}^{\prime}\left(\nu_{4}^{n}\right) \partial_{t}(\xi+\alpha)^{n}+\left[K_{s}^{\prime}\left(\nu_{3}^{n}\right)-K_{s}^{\prime}\left(\nu_{4}^{n}\right)\right] \partial_{t} \psi_{s}^{n}\right| \\
& \quad \leq Q\left\{\left|\partial_{t} \xi^{n}\right|+\left|\partial_{t} \alpha^{n}\right|+\left|\xi^{n}\right|+\left|\xi^{n-1}\right|+\left|\alpha^{n}\right|+\left|\alpha^{n-1}\right|+\Delta t^{n}\right\}
\end{aligned}
$$

where $Q$ depends on bounds for $K_{s}$ and $\partial \psi_{s} / \partial t$. Now

$$
\begin{aligned}
\sum_{n=1}^{\bar{n}} & \left(K_{s}\left(\Psi_{s}^{n}\right), \partial_{t}\left(\left[\frac{\partial \xi}{\partial z}\right]^{2}\right)^{n}\right) \Delta t^{n} \\
= & \left(K_{s}\left(\Psi_{s}^{\bar{n}}\right) \frac{\partial \xi^{\bar{n}}}{\partial z}, \frac{\partial \xi^{\bar{n}}}{\partial z}\right)-\left(K_{s}\left(\Psi_{s}^{1}\right) \frac{\partial \xi^{0}}{\partial z}, \frac{\partial \xi^{0}}{\partial z}\right) \\
& -\sum_{n=1}^{\bar{n}-1}\left(\partial_{t}\left(K_{s}\left(\Psi_{s}\right)-K_{s}\left(\psi_{s}\right)\right)^{n+1},\left(\frac{\partial \xi^{n}}{\partial z}\right)^{2}\right) \Delta t^{n+1} \\
& -\sum_{n=1}^{\bar{n}-1}\left(\partial_{t}\left(K_{s}\left(\psi_{s}\right)\right)^{n+1},\left(\frac{\partial \xi^{n}}{\partial z}\right)^{2}\right) \Delta t^{n+1}
\end{aligned}
$$


and the first two and the last terms are easily controlled, while the third term will require a bit more work (see (4.26)-(4.27) below). Also,

$$
\begin{aligned}
\sum_{n=1}^{\bar{n}} & \left(\left[K_{s}\left(\psi_{s}^{n}\right)-K_{s}\left(\Psi_{s}^{n}\right)\right]\left(\frac{\partial \tilde{\Psi}_{s}^{n}}{\partial z}-g\right), \partial_{t}\left(\frac{\partial \xi}{\partial z}\right)^{n}\right) \Delta t^{n} \\
= & \left(\left[K_{s}\left(\psi_{s}^{\bar{n}}\right)-K_{s}\left(\Psi_{s}^{\bar{n}}\right)\right]\left(\frac{\partial \tilde{\Psi}_{s}^{\bar{n}}}{\partial z}-g\right), \frac{\partial \xi^{\bar{n}}}{\partial z}\right) \\
& -\left(\left[K_{s}\left(\psi_{s}^{1}\right)-K_{s}\left(\Psi_{s}^{1}\right)\right]\left(\frac{\partial \tilde{\Psi}_{s}^{1}}{\partial z}-g\right), \frac{\partial \xi^{0}}{\partial z}\right) \\
& -\sum_{n=1}^{\bar{n}-1}\left(\partial_{t}\left[K_{s}\left(\psi_{s}\right)-K_{s}\left(\Psi_{s}\right)\right]^{n+1}\left(\frac{\partial \tilde{\Psi}_{s}^{n+1}}{\partial z}-g\right), \frac{\partial \xi^{n}}{\partial z}\right) \Delta t^{n+1} \\
& +\sum_{n=1}^{\bar{n}-1}\left(\left[K_{s}\left(\psi_{s}^{n}\right)-K_{s}\left(\Psi_{s}^{n}\right)\right] \partial_{t}\left(\frac{\partial \tilde{\Psi}_{s}^{n}}{\partial z}\right)^{n+1}, \frac{\partial \xi^{n}}{\partial z}\right) \Delta t^{n+1},
\end{aligned}
$$

and each term is easily controlled therein.

Note that the term containing $q_{\text {evap }}$ is controlled by Lemma 2 :

$$
\begin{aligned}
& {\left[q_{\text {evap }}^{n}\left(\Psi_{s}^{n}(0)\right)-q_{\text {evap }}^{n}\left(\psi_{s}^{n}(0)\right)\right] \partial_{t} \xi^{n}(0)} \\
& \geq \partial_{t}\left(\int_{\Psi_{s}(0)}^{\psi_{s}(0)}\left[q_{\text {evap }}\left(\psi_{s}(0)\right)-q_{\text {evap }}(\mu)\right] d \mu\right)^{n} \\
& \quad-Q\left\{\left(\xi^{n}(0)\right)^{2}+\left(\xi^{n-1}(0)\right)^{2}+\left(\alpha^{n}(0)\right)^{2}\right. \\
& \left.\quad+\left(\alpha^{n-1}(0)\right)^{2}+\left(\Delta t^{n}\right)^{2}+\left(\partial_{t} \alpha^{n}(0)\right)^{2}\right\}
\end{aligned}
$$

where $Q$ depends on bounds for $q_{\text {evap }}$ and $\partial \psi_{s} / \partial t$.

Multiplying (4.19) through by $\Delta t^{n}$ and summing up to $\bar{n}$, we see from (4.20)-(4.24) and (4.11) that with $\Delta t^{0} \equiv \Delta t^{1}$ and $\alpha^{-1}=\alpha^{0}$,

$$
\begin{aligned}
\sum_{n=1}^{\bar{n}} & \left\|\partial_{t} \xi^{n}\right\|_{0}^{2} \Delta t^{n}+\left\|\frac{\partial \xi^{\bar{n}}}{\partial z}\right\|_{0}^{2}+\int_{\Psi_{s}^{n}(0)}^{\psi_{0}^{\bar{n}}(0)}\left[q_{\text {evap }}^{\bar{n}}\left(\psi_{s}^{\bar{n}}(0)\right)-q_{\text {evap }}^{\bar{n}}(\mu)\right] d \mu \\
\leq & Q\left\{\max _{n}\left[\left\|\xi^{n}\right\|_{0}^{2}+\left\|\alpha^{n}\right\|_{0}^{2}\right]+\left\|\xi^{0}\right\|_{1}^{2}+(\Delta t)^{2}\right. \\
& +\sum_{n=0}^{\bar{n}}\left[\left\|\xi^{n}\right\|_{1}^{2}+\left(\alpha^{n}(0)\right)^{2}+\left(\partial_{t} \alpha^{n}(0)\right)^{2}+\left\|\partial_{t} \alpha^{n}\right\|_{0}^{2}+\left\|\beta^{n}\right\|_{0}^{2}\right] \Delta t^{n} \\
& \left.+\sum_{n=1}^{\bar{n}-1}\left\|\left(\frac{\partial \xi^{n}}{\partial z}\right)^{2}\right\|_{0}^{2} \Delta t^{n}\right\}
\end{aligned}
$$

where $Q$ depends on bounds for $\rho, \theta, q_{\text {evap }}, S, A_{s}, A_{x}, \bar{K}_{x}, K_{s}, \partial \psi_{s} / \partial t, \partial^{2} \psi_{s} / \partial t^{2}$, $\partial \tilde{\Psi}_{s} / \partial t, \partial^{2} \tilde{\Psi}_{s} / \partial t \partial z, g$, and $\Delta t^{n+1} / \Delta t^{n}$. For the last term above, we need to use the inverse inequality and an induction argument. First we conclude that

$$
\left\|\left(\frac{\partial \xi^{n}}{\partial z}\right)^{2}\right\|_{0}^{2}=\left\|\frac{\partial \xi^{n}}{\partial z}\right\|_{L^{4}(I)}^{4} \leq Q\left\|\frac{\partial \xi^{n}}{\partial z}\right\|_{0}^{4} h^{-1} .
$$


Now we assume that for some fixed $C$,

$$
\left\|\frac{\partial \xi^{n}}{\partial z}\right\|_{0} \leq C h^{1 / 2} \text { for all } n<\bar{n}
$$

this vacuously holds for $\bar{n}=1$. Then we can write an inequality which has the same form as (4.25) without its last term, where now $Q$ depends also on $C$. With Theorem 2 and (4.17), we obtain parts (iii) and (v) of Theorem 1 with $N$ replaced by $\bar{n}$. But now for $h$ and $\Delta t$ sufficiently small, we obtain (4.27) for $n=\bar{n}$ and the induction can be continued.

As a final remark in this section, we note that (4.1) can be used to initialize our scheme. If $\Psi_{s}^{0}=\tilde{\Psi}_{s}^{0}$ (i.e., $\xi^{0}=0$ ), then $(4.25)$ simplifies and we can replace " $q-1$ " by " $q$ " in part (v) of Theorem 1 .

Corollary 1. Assume (A1)-(A9). If $\Psi_{s}^{0}=\tilde{\Psi}_{s}^{0}$, then for $h$ and $\Delta t$ sufficiently small and for any $1 \leq q \leq p$,

$$
\begin{aligned}
\left(\sum_{n=1}^{N}\left\|\partial_{t}\left(\Psi_{s}-\psi_{s}\right)^{n}\right\|_{0}^{2} \Delta t^{n}\right)^{1 / 2} \leq C\{[ & \left(\sum_{n=1}^{N}\left\|\frac{\partial \psi_{s}^{n}}{\partial t}\right\|_{q}^{2} \Delta t^{n}\right)^{1 / 2}+\left\|\psi_{s}\right\|_{L^{\infty}\left(J_{;} H^{q}(I)\right)} \\
& \left.\left.+\left\|\psi_{x}\right\|_{L^{\infty}\left(J ; H^{q}(I)\right)}+\left\|\psi_{s}^{0}\right\|_{q}\right] h^{q}+\Delta t\right\}
\end{aligned}
$$

where $C$ is independent of $h$ and $\Delta t$.

5. Calculation of the water flow rate. One of the important quantities of interest to the agricultural scientist is the water flow rate up the taproot, which we denote by $\gamma_{x}$ :

$$
\gamma_{x}=\bar{K}_{x}\left(\frac{\partial \psi_{x}}{\partial z}-g\right)
$$

From Theorem 1, part (iv), we know that this quantity is accurate to order $p-1$ in $h$ and order 1 in $\Delta t$ in the $L^{2}(I)$-norm in space (at each time level). We would like the error to be expressed at each point of space, or at least at each of the $z_{i}$ 's. By an inverse inequality, we can obtain such an error estimate, at the cost of $h^{-1 / 2}$ multiplying the error. In this section we show how to approximate $\gamma_{x}$ at each time level and at each $z_{i}$ to the optimal order $p$ in $h$ and 1 in $\Delta t$.

For $i \geq 1$, we multiply (2.5) by $z-z_{i-1}$ and integrate in space over $\left(z_{i-1}, z_{i}\right)$ to obtain with (2.13) that

$$
\gamma_{x}\left(z_{i}, t\right) h_{i}=\int_{z_{i-1}}^{z_{i}} \bar{K}_{x}\left(\frac{\partial \psi_{x}}{\partial z}-g\right) d z-\int_{z_{i-1}}^{z_{i}} A_{x}^{-1} S\left(\psi_{s}-\psi_{x}\right)\left(z-z_{i-1}\right) d z
$$

This motivates the definition [8], [17] of our improved approximate flux $\Gamma_{x, i}^{n}$ :

$$
\Gamma_{x, i}^{n} h_{i}=\int_{z_{i-1}}^{z_{i}} \bar{K}_{x}\left(\frac{\partial \Psi_{x}^{n}}{\partial z}-g\right) d z-\int_{z_{i-1}}^{z_{i}} A_{x}^{-1} S^{n}\left(\Psi_{s}^{n}-\Psi_{x}^{n}\right)\left(z-z_{i-1}\right) d z
$$

This quantity can be computed easily from the approximate solution. 
We now consider the error in this flux approximation. From (5.2)-(5.3),

$$
\begin{aligned}
\left(\Gamma_{x, i}^{n}-\gamma_{x}\left(z_{i}, t^{n}\right)\right) h_{i}= & \int_{z_{i-1}}^{z_{i}} \bar{K}_{x}\left(\frac{\partial \Psi_{x}^{n}}{\partial z}-\frac{\partial \psi_{x}^{n}}{\partial z}\right) d z \\
& -\int_{z_{i-1}}^{z_{i}} A_{x}^{-1} S^{n}\left(\left(\Psi_{s}^{n}-\psi_{s}^{n}\right)-\left(\Psi_{x}^{n}-\psi_{x}^{n}\right)\right)\left(z-z_{i-1}\right) d z
\end{aligned}
$$

For $i=1,2, \ldots, M$, let

$$
\phi_{i}(z)= \begin{cases}0 & \text { if } 0 \leq z \leq z_{i-1} \\ z-z_{i-1} & \text { if } z_{i-1} \leq z \leq z_{i} \\ h_{i} & \text { if } z_{i} \leq z \leq L\end{cases}
$$

and note that $\phi_{i} \in \mathcal{U}_{h}$. Take $\phi_{i}$ in both (3.4) and (3.2); the difference is then

$$
\left(\bar{K}_{x}\left(\frac{\partial \Psi_{x}^{n}}{\partial z}-\frac{\partial \psi_{x}^{n}}{\partial z}\right), \frac{\partial \phi_{i}}{\partial z}\right)=\left(A_{x}^{-1} S^{n}\left(\left(\Psi_{s}^{n}-\psi_{s}^{n}\right)-\left(\Psi_{x}^{n}-\psi_{x}^{n}\right)\right), \phi_{i}\right)
$$

'This further implies that

$$
\begin{aligned}
& \left(\Gamma_{x, i}^{n}-\gamma_{x}\left(z_{i}, t^{n}\right)\right) h_{i} \\
& =\left(A_{x}^{-1} S^{n}\left(\left(\Psi_{s}^{n}-\psi_{s}^{n}\right)-\left(\Psi_{x}^{n}-\psi_{x}^{n}\right)\right), \phi_{i}\right) \\
& \quad-\int_{z_{i-1}}^{z_{i}} A_{x}^{-1} S^{n}\left(\left(\Psi_{s}^{n}-\psi_{s}^{n}\right)-\left(\Psi_{x}^{n}-\psi_{x}^{n}\right)\right)\left(z-z_{i-1}\right) d z \\
& =h_{i} \int_{z_{i}}^{L} A_{x}^{-1} S^{n}\left(\left(\Psi_{s}^{n}-\psi_{s}^{n}\right)-\left(\Psi_{x}^{n}-\psi_{x}^{n}\right)\right) d z
\end{aligned}
$$

and hence,

$$
\left|\Gamma_{x, i}^{n}-\gamma_{x}\left(z_{i}, t^{n}\right)\right| \leq Q\left\{\left\|\Psi_{s}^{n}-\psi_{s}^{n}\right\|_{L^{1}(I)}+\left\|\Psi_{x}^{n}-\psi_{x}^{n}\right\|_{L^{1}(I)}\right\}
$$

where $Q$ depends on bounds for $S$ and $A_{x}^{-1}$.

In an entirely similar way, we may also approximate the water flow rate up the soil column. We define

$$
\gamma_{s}=K_{s}\left(\psi_{s}\right)\left(\frac{\partial \psi_{s}}{\partial z}-g\right)
$$

and we note that for $i \geq 1$, if we multiply (2.1) by $z-z_{i-1}$ and integrate in space over $\left(z_{i-1}, z_{i}\right)$, we obtain with $(2.13)$ that

$$
\begin{aligned}
\gamma_{s}\left(z_{i}, t\right) h_{i}= & \int_{z_{i-1}}^{z_{i}} \rho \frac{\partial \theta\left(\psi_{s}\right)}{\partial t}\left(z-z_{i-1}\right) d z+\int_{z_{i-1}}^{z_{i}} K_{s}\left(\psi_{s}\right)\left(\frac{\partial \psi_{s}}{\partial z}-g\right) d z \\
& +\int_{z_{i-1}}^{z_{i}} A_{s}^{-1} S\left(\psi_{s}-\psi_{x}\right)\left(z-z_{i-1}\right) d z
\end{aligned}
$$

Therefore we define our improved approximate flux $\Gamma_{s, i}^{n}$ by

$$
\begin{aligned}
\Gamma_{s, i}^{n} h_{i}= & \int_{z_{i-1}}^{z_{i}} \rho \partial_{t} \theta\left(\Psi_{s}\right)^{n}\left(z-z_{i-1}\right) d z+\int_{z_{i-1}}^{z_{i}} K_{s}\left(\Psi_{s}^{n}\right)\left(\frac{\partial \Psi_{s}^{n}}{\partial z}-g\right) d z \\
& +\int_{z_{i-1}}^{z_{i}} A_{s}^{-1} S^{n}\left(\Psi_{s}^{n}-\Psi_{x}^{n}\right)\left(z-z_{i-1}\right) d z .
\end{aligned}
$$


For the error, we first note that

$$
\begin{aligned}
\left(\Gamma_{s, i}^{n}-\gamma_{s}\left(z_{i}, t^{n}\right)\right) h_{i} \\
=\int_{z_{i-1}}^{z_{i}} \rho\left(\partial_{t} \theta\left(\Psi_{s}\right)^{n}-\partial_{t} \theta\left(\psi_{s}\right)^{n}\right)\left(z-z_{i-1}\right) d z \\
\quad+\int_{z_{i-1}}^{z_{i}}\left[K_{s}\left(\Psi_{s}^{n}\right)\left(\frac{\partial \Psi_{s}^{n}}{\partial z}-g\right)-K_{s}\left(\psi_{s}^{n}\right)\left(\frac{\partial \psi_{s}^{n}}{\partial z}-g\right)\right] d z \\
\quad+\int_{z_{i-1}}^{z_{i}} A_{s}^{-1} S^{n}\left(\left(\Psi_{s}^{n}-\psi_{s}^{n}\right)-\left(\Psi_{x}^{n}-\psi_{x}^{n}\right)\right)\left(z-z_{i-1}\right) d z \\
\quad+\int_{z_{i-1}}^{z_{i}} \rho\left(\partial_{t} \theta\left(\psi_{s}\right)^{n}-\frac{\partial \theta\left(\psi_{s}^{n}\right)}{\partial t}\right)\left(z-z_{i-1}\right) d z
\end{aligned}
$$

Taking $\phi_{i}-z h_{i} / L \in \mathcal{V}_{h}$ in (4.9), we obtain

$$
\begin{aligned}
\left(\rho\left[\partial_{t} \theta\left(\Psi_{s}\right)^{n}-\partial_{t} \theta\left(\psi_{s}\right)^{n}\right], \phi_{i}-z h_{i} / L\right) \\
\quad+\left(K_{s}\left(\Psi_{s}^{n}\right)\left(\frac{\partial \Psi_{s}^{n}}{\partial z}-g\right)-K_{s}\left(\psi_{s}^{n}\right)\left(\frac{\partial \tilde{\Psi}_{s}^{n}}{\partial z}-g\right), \frac{\partial \phi_{i}}{\partial z}-\frac{h_{i}}{L}\right) \\
=-\left(A_{s}^{-1} S^{n}\left[\xi^{n}-\left(\eta^{n}+\beta^{n}\right)\right], \phi_{i}-z h_{i} / L\right) \\
\quad-\left(\rho\left[\partial_{t} \theta\left(\psi_{s}\right)^{n}-\frac{\partial \theta\left(\psi_{s}^{n}\right)}{\partial t}\right], \phi_{i}-z h_{i} / L\right),
\end{aligned}
$$

and this combined with (5.12) yields that

$$
\begin{aligned}
\left(\Gamma_{s, i}^{n}-\right. & \left.\gamma_{s}\left(z_{i}, t^{n}\right)\right) h_{i} \\
= & -\int_{0}^{L} \rho\left(\partial_{t} \theta\left(\Psi_{s}\right)^{n}-\partial_{t} \theta\left(\psi_{s}\right)^{n}\right)\left(h_{i} \chi_{\left(z_{i}, L\right)}-z h_{i} / L\right) d z \\
& +\frac{h_{i}}{L} \int_{0}^{L}\left[K_{s}\left(\Psi_{s}^{n}\right)\left(\frac{\partial \Psi_{s}^{n}}{\partial z}-g\right)-K_{s}\left(\psi_{s}^{n}\right)\left(\frac{\partial \tilde{\Psi}_{s}^{n}}{\partial z}-g\right)\right] d z \\
& -\int_{0}^{L} A_{s}^{-1} S^{n}\left(\left(\Psi_{s}^{n}-\psi_{s}^{n}\right)-\left(\Psi_{x}^{n}-\psi_{x}^{n}\right)\right)\left(h_{i} \chi_{\left(z_{i}, L\right)}-z h_{i} / L\right) d z \\
& -\int_{0}^{L} \rho\left(\partial_{t} \theta\left(\psi_{s}\right)^{n}-\frac{\partial \theta\left(\psi_{s}^{n}\right)}{\partial t}\right)\left(h_{i} \chi_{\left(z_{i}, L\right)}-z h_{i} / L\right) d z \\
& +\int_{z_{i-1}}^{z_{i}} K_{s}\left(\psi_{s}^{n}\right)\left(\frac{\partial \tilde{\Psi}_{s}^{n}}{\partial z}-\frac{\partial \psi_{s}^{n}}{\partial z}\right) d z,
\end{aligned}
$$

where $\chi_{\left(z_{i}, L\right)}(z)$ is the characteristic function of $\left(z_{i}, L\right)$. The last term can be integrated by parts,

$$
\begin{aligned}
& \int_{z_{i-1}}^{z_{i}} K_{s}\left(\psi_{s}^{n}\right)\left(\frac{\partial \tilde{\Psi}_{s}^{n}}{\partial z}-\frac{\partial \psi_{s}^{n}}{\partial z}\right) d z \\
& \quad=K_{s}\left(\psi_{s}^{n}\left(z_{i}\right)\right)\left(\tilde{\Psi}_{s}^{n}\left(z_{i}\right)-\psi_{s}^{n}\left(z_{i}\right)\right)-K_{s}\left(\psi_{s}^{n}\left(z_{i-1}\right)\right)\left(\tilde{\Psi}_{s}^{n}\left(z_{i-1}\right)-\psi_{s}^{n}\left(z_{i-1}\right)\right) \\
& \quad-\int_{z_{i-1}}^{z_{i}} K_{s}^{\prime}\left(\psi_{s}^{n}\right) \frac{\partial \psi_{s}^{n}}{\partial z}\left(\tilde{\Psi}_{s}^{n}-\psi_{s}^{n}\right) d z
\end{aligned}
$$


Then

$$
\begin{aligned}
&\left|\Gamma_{s, i}^{n}-\gamma_{s}\left(z_{i}, t^{n}\right)\right| \\
& \leq Q\left\{\left\|\partial_{t} \theta\left(\Psi_{s}\right)^{n}-\partial_{t} \theta\left(\psi_{s}\right)^{n}\right\|_{L^{1}(I)}+\left\|\partial_{t} \theta\left(\psi_{s}\right)^{n}-\frac{\partial \theta\left(\psi_{s}\right)}{\partial t}\right\|_{L^{1}(I)}\right. \\
&+\left\|\Psi_{s}^{n}-\psi_{s}^{n}\right\|_{L^{1}(I)}+\left\|\Psi_{x}^{n}-\psi_{x}^{n}\right\|_{L^{1}(I)}+\left\|\tilde{\Psi}_{s}^{n}-\Psi_{s}^{n}\right\|_{1} \\
&\left.+\left\|\tilde{\Psi}_{s}^{n}-\psi_{s}^{n}\right\|_{0}+\left|\tilde{\Psi}_{s}^{n}\left(z_{i}\right)-\psi_{s}^{n}\left(z_{i}\right)\right|+\left|\tilde{\Psi}_{s}^{n}\left(z_{i-1}\right)-\psi_{s}^{n}\left(z_{i-1}\right)\right|\right\}
\end{aligned}
$$

where $Q$ depends on $\rho, K_{s}, K_{s}^{\prime},\left\|\partial \tilde{\Psi}_{s} / \partial z\right\|_{L^{\infty}\left(J ; L^{\infty}(I)\right)},\left\|\partial \psi_{s} / \partial z\right\|_{L^{\infty}\left(J ; L^{\infty}(I)\right)}, S$, and $A_{3}$, and so finally,

$$
\begin{aligned}
\left(\sum_{n=1}^{N}\right. & \left.\left|\Gamma_{s, i}^{n}-\gamma_{s}\left(z_{i}, t^{n}\right)\right|^{2} \Delta t^{n}\right)^{1 / 2} \\
\leq & Q\left\{\left(\sum_{n=1}^{N}\left\|\partial_{t}\left(\Psi_{s}-\psi_{s}\right)^{n}\right\|_{L^{1}(I)}^{2} \Delta t^{n}\right)^{1 / 2}+\Delta t\right. \\
& +\max _{n}\left[\left\|\Psi_{s}^{n}-\psi_{s}^{n}\right\|_{L^{1}(I)}+\left\|\Psi_{x}^{n}-\psi_{x}^{n}\right\|_{L^{1}(I)}+\left\|\tilde{\Psi}_{s}^{n}-\Psi_{s}^{n}\right\|_{1}\right. \\
& \left.\left.+\left\|\tilde{\Psi}_{s}^{n}-\psi_{s}^{n}\right\|_{0}+\left|\tilde{\Psi}_{s}^{n}\left(z_{i}\right)-\psi_{s}^{n}\left(z_{i}\right)\right|+\left|\tilde{\Psi}_{s}^{n}\left(z_{i-1}\right)-\psi_{s}^{n}\left(z_{i-1}\right)\right|\right]\right\}
\end{aligned}
$$

where now $Q$ depends also on $\theta^{\prime}$ and $\left\|\partial^{2} \theta\left(\psi_{s}\right) / \partial t^{2}\right\|_{L^{1}(I)}$ after using an expression similar to (4.14).

Theorem 1 and Corollary 1 combined with (5.8), (5.17), (4.25), and Theorem 2 shows that these improved discrete fluxes are superconvergent approximations to the true fluxes.

Theorem 3. Assume (A1)-(A9). Then for some constant $C$ depending on $u$ and its derivatives, but not on $h, \Delta t, t, z_{i}$, or $n$, and for $h$ and $\Delta t$ sufficiently small,

(i) $\max _{n}\left|\Gamma_{x, i}^{n}-\gamma_{x}\left(z_{i}, t^{n}\right)\right| \leq C\left\{h^{p}+\Delta t\right\}$

(ii) $\left(\sum_{n=1}^{N}\left|\Gamma_{s, i}^{n}-\gamma_{s}\left(z_{i}, t^{n}\right)\right|^{2} \Delta t^{n}\right)^{1 / 2} \leq C\left\{h^{p-1}+\Delta t\right\}$,

for any $i=1, \ldots, M$. Moreover, if $\Psi_{s}^{0}=\tilde{\Psi}_{s}^{0}$, then $p$ replaces $p-1$ in (ii).

6. A finite difference version of the scheme. We define a finite difference scheme as a special case of our finite element scheme by specifying a certain choice for the $\mathcal{V}_{h}$ and $\mathcal{U}_{h}$, and by requiring certain quadrature rules in the evaluation of the integrals. We take as finite dimensional subspaces the continuous, piece-wise linear functions defined on the given mesh; that is, $\mathcal{V}_{h}=\operatorname{span}\left\{w_{i}\right\}_{i=0}^{M-1}$ and $\mathcal{U}_{h}=$ $\operatorname{span}\left\{w_{i}\right\}_{i=0}^{M}$, where

$$
w_{i}(z)= \begin{cases}\left(z-z_{i-1}\right) / h_{i} & \text { if } z_{i-1} \leq z \leq z_{i} \\ \left(z_{i+1}-z\right) / h_{i+1} & \text { if } z_{i} \leq z \leq z_{i+1} \\ 0 & \text { otherwise }\end{cases}
$$

We use the midpoint rule to evaluate any integral involving a spatial derivative, and otherwise we use the trapezoidal rule. We denote these rules, respectively, as discrete 
inner products for any $u$ and $v$ as

$$
\langle u, v\rangle_{\mathrm{M}}=\sum_{i=1}^{M} u_{i-1 / 2} v_{i-1 / 2} h_{i}, \quad \text { and } \quad\langle u, v\rangle_{\mathrm{T}}=\frac{1}{2} \sum_{i=1}^{M}\left(u_{i} v_{i}+u_{i-1} v_{i-1}\right) h_{i},
$$

where we use the notation $u_{i}=u\left(z_{i}\right), z_{i+1 / 2}=\left(z_{i}+z_{i+1}\right) / 2$, and $u_{i+1 / 2}=u\left(z_{i+1 / 2}\right)$. Note that for $u \in \mathcal{U}_{h}$ or $\mathcal{V}_{h}, u_{i+1 / 2}=\left(u_{i}+u_{i+1}\right) / 2$.

Our finite difference procedure is then the following: For $n=1, \ldots, N$, let $\Psi_{s}^{n} \in$ $\mathcal{V}_{h}+\psi_{\text {bot }}^{n}$ and $\Psi_{x}^{n} \in \mathcal{U}_{h}$ satisfy

$$
\begin{gathered}
\left\langle\rho \partial_{t} \theta\left(\Psi_{s}\right)^{n}, w_{i}\right\rangle_{\mathrm{T}}+\left\langle K_{s}\left(\Psi_{s}^{n}\right)\left(\frac{\partial \Psi_{s}^{n}}{\partial z}-g\right), \frac{\partial w_{i}}{\partial z}\right\rangle_{\mathrm{M}} \\
=-\left[q_{\text {evap }}^{n}\left(\Psi_{s}^{n}(0)\right)-q_{\mathrm{prec}}^{n}\right] \delta_{i, 0}-\left\langle A_{s}^{-1} S^{n}\left(\Psi_{s}^{n}-\Psi_{x}^{n}\right), w_{i}\right\rangle_{\mathrm{T}} \\
\text { for } i=0,1, \ldots, M-1,
\end{gathered}
$$

and

$$
\begin{array}{r}
\left\langle\bar{K}_{x}\left(\frac{\partial \Psi_{x}^{n}}{\partial z}-g\right), \frac{\partial w_{i}}{\partial z}\right\rangle_{\mathrm{M}}=-A_{x}^{-1} q_{\mathrm{trans}}^{n} \delta_{i, 0}+\left\langle A_{x}^{-1} S^{n}\left(\Psi_{s}^{n}-\Psi_{x}^{n}\right), w_{i}\right\rangle_{\mathrm{T}} \\
\text { for } i=0,1, \ldots, M
\end{array}
$$

where $\delta_{i, j}$ is the Kronecker delta. We initialize the scheme by choosing the interpolant of $\psi_{s}^{0}$ :

$$
\Psi_{s, i}^{0}=\psi_{s, i}^{0} \quad \text { for } i=0,1, \ldots, M .
$$

Our scheme can be expressed equivalently in more traditional finite difference notation as follows. We first define by reflection $h_{0}=h_{1}, h_{M+1}=h_{M}$,

$$
\begin{gathered}
\psi_{s,-1}=\psi_{s, 1}-2 g h_{1}, \quad \psi_{x,-1}=\psi_{x, 1}-2 g h_{1}, \quad \psi_{x, M+1}=\psi_{x, M-1}+2 g h_{M}, \\
\text { and } K_{s}\left(\Psi_{s,-1 / 2}^{n}\right)=K_{s}\left(\Psi_{s, 1 / 2}^{n}\right) .
\end{gathered}
$$

Then, for $n=1, \ldots, N$ and $i=0, \ldots, M$, let $\Psi_{s, i}^{n}$ and $\Psi_{x, i}^{n}$ satisfy

$$
\begin{aligned}
& \Psi_{s, i}^{0}=\psi_{s, i}^{0}, \\
& \Psi_{s, M}^{n}=\psi_{\text {bot }}^{n}, \\
& \rho \partial_{t} \theta\left(\Psi_{s, i}\right)^{n}-\frac{2}{h_{i+1}+h_{i}}\left[K_{s}\left(\Psi_{s, i+1 / 2}^{n}\right)\left(\frac{\Psi_{s, i+1}^{n}-\Psi_{s, i}^{n}}{h_{i+1}}-g\right)\right. \\
& \left.-K_{s}\left(\Psi_{s, i-1 / 2}^{n}\right)\left(\frac{\Psi_{s, i}^{n}-\Psi_{s, i-1}^{n}}{h_{i}}-g\right)\right] \\
& =-\frac{2}{h_{1}}\left[q_{\text {evap }}^{n}\left(\Psi_{s, 0}^{n}\right)-q_{\mathrm{prec}}^{n}\right] \delta_{i, 0}-A_{s}^{-1} S_{i}^{n}\left(\Psi_{s, i}^{n}-\Psi_{x, i}^{n}\right) \quad \text { if } i \neq M, \\
& -\frac{2 \bar{K}_{x}}{h_{i+1}+h_{i}}\left(\frac{\Psi_{x, i+1}^{n}-\Psi_{x, i}^{n}}{h_{i+1}}-\frac{\Psi_{x, i}^{n}-\Psi_{x, i-1}^{n}}{h_{i}}\right) \\
& =-\frac{2}{h_{1}} A_{x}^{-1} q_{\mathrm{trans}}^{n} \delta_{i, 0}+A_{x}^{-1} S_{i}^{n}\left(\Psi_{s, i}^{n}-\Psi_{x, i}^{n}\right) .
\end{aligned}
$$


We can also define the improved fluxes by the same choice of quadrature rules: the midpoint rule on integrals involving spatial derivatives and the trapezoidal rule otherwise. From (5.3) and (5.11), we therefore define for $i=1, \ldots, M$,

$$
\begin{aligned}
\Gamma_{x, i}^{n}= & \bar{K}_{x}\left(\frac{\Psi_{x, i}-\Psi_{x, i-1}}{h_{i}}-g\right)-\frac{1}{2} A_{x}^{-1} S_{i}^{n}\left(\Psi_{s, i}-\Psi_{x, i}\right) h_{i} \\
\Gamma_{s, i}^{n}= & \frac{1}{2} \rho \partial_{t} \theta\left(\Psi_{s, i}\right)^{n} h_{i}+K_{s}\left(\Psi_{s, i-1 / 2}^{n}\right)\left(\frac{\Psi_{s, i}-\Psi_{s, i-1}}{h_{i}}-g\right) \\
& +\frac{1}{2} A_{s}^{-1} S_{i}^{n}\left(\Psi_{s, i}-\Psi_{x, i}\right) h_{i} .
\end{aligned}
$$

An analysis of the error in a system similar to (6.1)-(6.3) was given by the authors in [3] (essentially the unreformulated model [15] was treated). A similar analysis can be given for (6.1)-(6.3). We first need to note several results. The error in approximating an integral by either the mid-point or the trapezoidal rule is of order $h^{2}[6]:$ For $R=\mathrm{M}$ or $\mathrm{T}$, if

$$
E_{R}(u, v) \equiv(u, v)-\langle u, v\rangle_{R}
$$

then

$$
\left|E_{R}(u, v)\right| \leq C_{\mathrm{E}} \sum_{i=1}^{M}\left\|(u v)^{\prime \prime}\right\|_{L^{1}\left(I_{i}\right)} h_{i}^{2}
$$

where $C_{\mathrm{E}}$ is independent of $u, v$, and the $h_{i}$. The error in the interpolant (6.3) is also of order $h^{2}$. Finally, all norms on a finite dimensional subspace are equivalent (see, in particular Lemma 1 of [3]). These facts, combined with the analysis of Sections 45 (see also [3]) allow us to obtain the following theorem analogous to Theorem 1, Corollary 1, and Theorem 3.

THEorem 4. Assume (A1)-(A9) with $p=2$. Assume also that $\psi_{s}$ and $\psi_{x}$ are continuous. Then for $h$ and $\Delta t$ sufficiently small,

(i) $\max _{n}\left\|\Psi_{s}^{n}-\psi_{s}^{n}\right\|_{0}+\max _{n}\left\|\Psi_{x}^{n}-\psi_{x}^{n}\right\|_{0} \leq C\left\{h^{2}+\Delta t\right\}$,

(ii) $\max _{n}\left\|\frac{\partial}{\partial z}\left(\Psi_{s}^{n}-\psi_{s}^{n}\right)\right\|_{0}^{n}+\max _{n}\left\|\frac{\partial}{\partial z}\left(\Psi_{x}^{n}-\psi_{x}^{n}\right)\right\|_{0} \leq C\{h+\Delta t\}$,

(iii) $\left(\sum_{n=1}^{N}\left\|\partial_{t}\left(\Psi_{s}-\psi_{s}\right)^{n}\right\|_{0}^{2} \Delta t^{n}\right)^{1 / 2} \leq C\{h+\Delta t\}$

(iv) $\max _{n}\left|\Gamma_{x, i}^{n}-\gamma_{x, i}^{n}\right| \leq C\left\{h^{2}+\Delta t\right\}$,

(v) $\left(\sum_{n=1}^{N}\left|\Gamma_{s, i}^{n}-\gamma_{s, i}^{n}\right|^{2} \Delta t^{n}\right)^{1 / 2} \leq C\{h+\Delta t\}$

where $C$ depends on the bounds in the assumptions but is independent of $h$ and $\Delta t$, and where $i=1, \ldots, M$. Moreover, if the scheme is initialized by setting $\Psi_{s}^{0} \in \mathcal{V}_{h}+\psi_{\text {bot }}^{0}$ such that

$$
\left\langle K_{s}\left(\psi_{s}^{0}\right)\left(\frac{\partial \Psi_{s}^{0}}{\partial z}-\frac{\partial \psi_{s}^{0}}{\partial z}\right), \frac{\partial w_{i}}{\partial z}\right\rangle_{\mathrm{M}}=0 \quad \text { for } i=0,1, \ldots, M-1
$$

then $h^{2}$ replaces $h$ in (iii) and (v). 
7. Some computational results. In this section we verify experimentally the convergence results of Theorem 4 for the finite difference scheme defined in Section 6 . The data used in the simulations is given in Table 1 , wherein $r_{t}$ is the radius of a single taproot xylem and $N_{t}$ is the total number of xylems in the soil block (thus $A_{x}=N_{t} \pi r_{t}^{2}$ ), and also $\mu$ is the water viscosity and $\bar{K}_{x}$ is defined from the HagenPoiseuille law for fluid flow through pipes (thus $\bar{K}_{x}=\rho r_{t}^{2} / 8 \mu$ ). Note that since the initial soil potential is linear, the initialization of our finite difference scheme is exact; consequently, we should expect the improved soil flux (6.9) to be accurate to order 2 in $h$.

TABLE 1.

Simulation parameters.

\begin{tabular}{c|c}
\hline Quantity & Value (in S.I. units) \\
\hline$T$ & 7200 \\
$L$ & 1 \\
$A_{s}$ & 4 \\
$r_{t}$ & .0005 \\
$N_{t}$ & 150 \\
$\mathcal{L}(z)$ & $10(1-z)+.0005$ \\
$R$ & $2 \times 10^{8}$ \\
$r_{e}$ & .00022 \\
$r_{c}$ & .005 \\
$r_{s}$ & .02 \\
$\bar{K}_{s}$ & 0.58 \\
$\bar{K}_{\mathrm{c}}$ & 150 \\
$q_{\mathrm{trans}}(t)$ & 0 \\
$q_{\text {evap }}$ & 0 \\
$q_{\text {prec }}$ & $\max (.0013 \sin (\pi t / 43200), 0)$ \\
$K_{s}(\psi)$ & $\left.13.56(-\psi / 2350)^{(-12.2 / 10.9)}-1.09\right] \times 10^{-7}$ \\
$\theta(\psi)$ & $-\ln (-\psi / 2350) / 10.9$ \\
$\psi_{s}^{0}(z)$ & $-709.8+9.8 z$ \\
$\psi_{\text {bot }}$ & -700 \\
$\rho$ & 1000 \\
$\mu$ & 9.8 \\
$g$ & \\
\hline
\end{tabular}

To estimate the errors, we computed a fine grid solution and assumed it to be exact. In each case, the rates of convergence were determined from a nonlinear least squares fit to the data from several relatively coarse simulations. In Tables 2-4 we present experimental rates of convergence for the error in approximating $\psi_{s}, \psi_{x}$, $\partial \psi_{s} / \partial z, \partial \psi_{s} / \partial z, \gamma_{x}$, and $\gamma_{s}$.

Table 2 shows the rates of convergence with respect to $h$; that is, we varied $h$ from $2^{-2} \mathrm{~m}$ to $2^{-6} \mathrm{~m}$ but held $\Delta t$ to a constant and negligible value $14.0625 \mathrm{~s}$. Table 3 shows the rates of convergence with respect to $\Delta t$. We varied $\Delta t$ from $1800 \mathrm{~s}$ to $112.5 \mathrm{~s}$ and held $h$ to the negligible value $2^{-9} \mathrm{~m}$. Finally, Table 4 shows the rates of convergence of the potentials when $h$ and $\Delta t$ both vary, $h$ from $2^{-3} \mathrm{~m}$ to $2^{-7} \mathrm{~m}$ and $\Delta t=\left(225 \times 2^{9} \mathrm{~s} / \mathrm{m}^{2}\right) h^{2}$. By way of comparison, the fine grid solution was computed with $h=2^{-9} \mathrm{~m}$ and with $\Delta t=14.0625 \mathrm{~s}$ for Tables $2-3$ and $\Delta t=7.03125 \mathrm{~s}$ for Table 4 . In all cases, the experimental rates of convergence agreed with the theoretical results of Theorem 4. 
TABLE 2 .

Error fit to $C h^{p}$ for $\Delta t$ negligible.

\begin{tabular}{c|c|c|c}
\hline Error quantity & $C$ & $p$ & Standard deviation \\
\hline $\max _{n}\left\|\Psi_{s}^{n}-\psi_{s}^{n}\right\|_{0}$ & 0.895129 & 2.039488 & $1.84032 \mathrm{E}-05$ \\
$\max _{n}\left\|\Psi_{x}^{n}-\psi_{x}^{n}\right\|_{0}$ & 2.973285 & 2.025740 & $5.91017 \mathrm{E}-04$ \\
$\max _{n}\left\|\frac{\partial}{\partial z}\left(\Psi_{s}^{n}-\psi_{s}^{n}\right)\right\|_{0}$ & 5.083447 & 1.023832 & $9.56546 \mathrm{E}-04$ \\
$\max _{n}\left\|\frac{\partial}{\partial z}\left(\Psi_{x}^{n}-\psi_{x}^{n}\right)\right\|_{0}$ & 0.061799 & 1.018302 & $2.81745 \mathrm{E}-05$ \\
$\max _{i, n}\left|\Gamma_{x, i}^{n}-\gamma_{x, i}^{n}\right|$ & $6.089464 \mathrm{E}-04$ & 1.936893 & $4.61138 \mathrm{E}-07$ \\
$\max _{i}\left(\sum_{n=1}^{N}\left|\Gamma_{s, i}^{n}-\gamma_{s, i}^{n}\right|^{2} \Delta t^{n}\right)^{1 / 2}$ & $1.862345 \mathrm{E}-04$ & 1.856296 & $3.02396 \mathrm{E}-08$ \\
\hline
\end{tabular}

TABLE 3.

Error fit to $C \Delta t^{p}$ for $h$ negligible.

\begin{tabular}{c|c|c|c}
\hline Error quantity & $C$ & $p$ & Standard deviation \\
\hline $\max _{n}\left\|\Psi_{s}^{n}-\psi_{s}^{n}\right\|_{0}$ & $5.710491 \mathrm{E}-04$ & 1.002882 & $4.60225 \mathrm{E}-03$ \\
$\max _{n}\left\|\Psi_{x}^{n}-\psi_{x}^{n}\right\|_{0}$ & $6.639860 \mathrm{E}-04$ & 1.001364 & $5.12440 \mathrm{E}-03$ \\
$\max _{n}\left\|\frac{\partial}{\partial z}\left(\Psi_{s}^{n}-\psi_{s}^{n}\right)\right\|_{0}$ & $9.012473 \mathrm{E}-04$ & 1.002677 & $7.27797 \mathrm{E}-03$ \\
$\max _{n}\left\|\frac{\partial}{\partial z}\left(\Psi_{x}^{n}-\psi_{x}^{n}\right)\right\|_{0}$ & $1.383027 \mathrm{E}-09$ & 1.000711 & $1.02714 \mathrm{E}-08$ \\
$\max _{i, n}\left|\Gamma_{x, i}^{n}-\gamma_{x, i}^{n}\right|$ & $6.728934 \mathrm{E}-08$ & 0.996905 & $5.13103 \mathrm{E}-07$ \\
$\max _{i}^{N}\left(\sum_{n=1}^{N}\left|\Gamma_{s, i}^{n}-\gamma_{s, i}^{n}\right|^{2} \Delta t^{n}\right)^{1 / 2}$ & $4.388742 \mathrm{E}-08$ & 1.095067 & $3.89498 \mathrm{E}-07$ \\
\hline
\end{tabular}

TABLE 4.

Error fit to $C h^{p}$ for $\Delta t$ proportional to $h^{2}$.

\begin{tabular}{c|c|c|c}
\hline Error quantity & $C$ & $p$ & Standard deviation \\
\hline $\max _{n}\left\|\Psi_{s}^{n}-\psi_{s}^{n}\right\|_{0}$ & 66.95623 & 1.995729 & $3.77153 \mathrm{E}-03$ \\
$\max _{n}\left\|\Psi_{x}^{n}-\psi_{x}^{n}\right\|_{0}$ & 81.28292 & 2.002657 & $4.09957 \mathrm{E}-03$ \\
$\max _{i, n}\left|\Gamma_{x, i}^{n}-\gamma_{x, i}^{n}\right|$ & $6.319264 \mathrm{E}-03$ & 1.963899 & $3.34806 \mathrm{E}-07$ \\
$\max _{i}\left(\sum_{n=1}^{N}\left|\Gamma_{s, i}^{n}-\gamma_{s, i}^{n}\right|^{2} \Delta t^{n}\right)^{1 / 2}$ & $1.431431 \mathrm{E}-02$ & 2.157266 & $2.86687 \mathrm{E}-07$ \\
\hline
\end{tabular}

\section{REFERENCES}

[1] R. M. Anderson, M. Obeyesekere, And D. Upchurch, Microscopic radial model for fiow in a root-soil system, (to appear).

[2] T. ARBOGAST, An error analysis for Galerkin approximations to an equation of mixed elliptic-parabolic type, (to appear). 
[3] T. ARBogast, M. ObeYESEKERE, AND M. F. W HeEler, Convergence analysis for simulating flow in root-soil systems, in Proceedings of the Seventh Conference on the Mathematics of Finite Elements and Applications (MAFELAP 1990), Brunel University, Academic Press, London, 1991, to appear.

[4] T. Arbogast, M. ObeYesekere, and M. F. Wheeler, Simulation of flow in root-soil systems, (to appear).

[5] P. Ciarlet, The Finite Element Method for Elliptic Problems, North-Holland, Amsterdam, 1978.

[6] P. J. Davis, Interpolation and Approximation, Dover Publications, New York, 1975.

[7] J. Douglas, JR., AND T. DUPONT, Galerkin approximations for the two point boundary problem using continuous, piecewise polynomial spaces, Numer. Math., 22 (1974), pp. 99-109.

[8] R. J. Dunn, IR., AND M. F. WheELER, Some collocation-Galerkin methods for two-point boundary value problems, SIAM J. Numer. Anal., 13 (1976), pp. 720-733.

[9] T. Dupont, G. Fairweather, AND J. P. Johnson, Three-level Galerkin methods for parabolic equations, SIAM J. Numer. Anal., 11 (1974), pp. 392-410.

[10] R. A. Freeze and J. A. Cherry, Groundwater, Prentice-Hall, Englewood Cliffs, New Jersey, 1979.

[11] Y.-S. JUNG AND H. M. TAYLOR, Differences in water uptake rates of soybean roots associated with time and depth, Soil Sci., 137 (1984), pp. 341-350.

[12] B. KLePper, R. W. RickMan, AND H. M. TAYLOR, Farm management and the function of field crop root systems, Agricultural Water Management, 7 (1983), pp. 115-141.

[13] J. J. LANDSBerg And N. D. Fowkes, Water movement through plant roots, Ann. Bot., 42 (1978), pp. 493-508.

[14] F. J. Molz And C. M. Peterson, Water transport from roots to soil, Agron. J., 68 (1976), pp. 901-904.

[15] M. OBEYESEKERE, A porous media problem arising in the study of water transport in soil and roots, Ph.D. Thesis, Texas Tech University, Lubbock, Texas, 1989.

[16] M. F. WHEELER, A priori $L_{2}$ errot estimates for Galerkin approximations to parabolic partial differential equations, SIAM J. Numer. Anal., 10 (1973), pp. 723-759.

[17] M. F. WHEELER, A Galerkin procedure for estimating the flux for two-point boundary value problems, SIAM J. Numer. Anal., 11 (1974), pp. 764-768. 